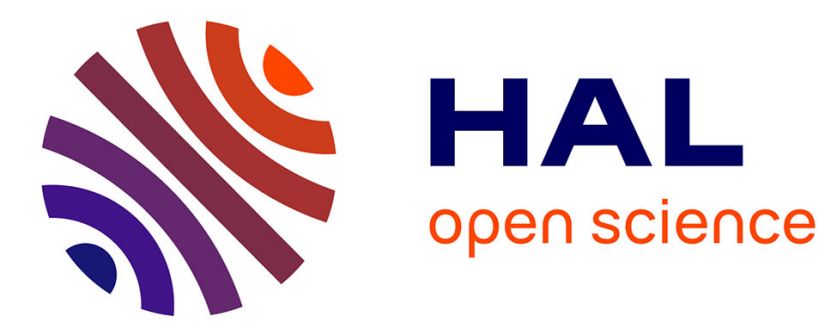

\title{
General Landau theory of non-symmetry-breaking and symmetry-breaking spin transition materials
}

Giovanni Azzolina, Roman Bertoni, Eric Collet

\section{To cite this version:}

Giovanni Azzolina, Roman Bertoni, Eric Collet. General Landau theory of non-symmetry-breaking and symmetry-breaking spin transition materials. Journal of Applied Physics, 2021, 129 (8), pp.085106. 10.1063/5.0041453 . hal-03157211

\section{HAL Id: hal-03157211 \\ https://hal.science/hal-03157211}

Submitted on 2 Mar 2021

HAL is a multi-disciplinary open access archive for the deposit and dissemination of scientific research documents, whether they are published or not. The documents may come from teaching and research institutions in France or abroad, or from public or private research centers.
L'archive ouverte pluridisciplinaire HAL, est destinée au dépôt et à la diffusion de documents scientifiques de niveau recherche, publiés ou non, émanant des établissements d'enseignement et de recherche français ou étrangers, des laboratoires publics ou privés. 


\title{
General Landau Theory of Non-Symmetry-Breaking and Symmetry-Breaking Spin Transition Materials
}

\author{
Giovanni Azzolina, ${ }^{1}$ Roman Bertoni, ${ }^{1}$ Eric Collet ${ }^{*, 1}$ \\ ${ }^{1}$ Univ Rennes, CNRS, IPR (Institut de Physique de Rennes) - UMR 6251, F-35000 Rennes, France \\ E-mail: eric.collet@univ-rennes1.fr
}

\begin{abstract}
Spin-transition materials, including the families of spin-crossover and charge-transfer systems, and more generally molecular-based materials exhibiting electronic and/or structural bistability, may undergo various types of phase transitions. The change of electronic state is stabilized by molecular reorganizations and both phenomena, which are usually non-symmetry breaking, can be described through the evolution of an order parameter $q$. Due to symmetry, $q$ linearly couples to volume change. It is known that such elastic interactions are responsible for cooperative phenomena in non-symmetry-breaking spin-transitions. However, spin-transition materials may also exhibit symmetry-breaking phenomena related to various types of orders such as structural order as well as spin-state concentration waves. The universal framework of the Landau theory of phase transition is relevant for describing such ordering processes through the evolution of a symmetry-breaking order parameter $\eta$. The simultaneous or sequential occurrence of spin transition and symmetry breaking phenomena are reported for numerous spin-transition materials and the coupling between these two types of instabilities is responsible for the emergence of various types of functions. In this work, we use the Landau approach to describe both symmetry-breaking phenomena and non-symmetry-breaking spin transition. We discuss how their coupling can generate sequences of phase transitions, from simple spin crossover to spin transition, continuous or discontinuous symmetry breaking, including ferroelasticity or stepwise spin transitions.
\end{abstract}




\section{Introduction}

Spin-transition materials, including the families of spin-crossover, ${ }^{1-3}$ charge-transfer (CT) systems $^{4-7}$, may undergo various types of phase transitions. The concept of molecular bistability expands to various types of systems exhibiting electronic and/or structural bistability between states of different entropy and volume. ${ }^{7-24}$ Phase transitions may be driven by external stimuli including temperature, pressure, magnetic field, electric field or light, allowing for the emergence of functions. In many cases, both the change of electronic state and the coupled structural reorganizations are non-symmetry breaking, which can be described through the evolution of an order parameter $q$. This order parameter $q$ transforms as the identity representation of the symmetry group and therefore linearly couples to the volume strain. That is the reason why cooperative phenomena in non-symmetry-breaking spin-transitions can occur, due to the relative change of the bonding or antibonding nature of the electronic distribution that couples to lattice expansion. Materials may also exhibit symmetry-breaking phenomena, related to diverse types of electronic and/or structural orders, including ferromagnetism, ferroelectricity or ferroelasticity. The universal Landau theory of phase transitions allows describing these symmetry-breaking phenomena through the evolution of one (or several) symmetry-breaking order parameter(s) $\eta$, the amplitude of which measures the deviation from the high symmetry phase.

The non-symmetry-breaking change of electronic state is an instability, which may couple to a symmetry-breaking phenomenon as recently highlighted. ${ }^{25}$ In this paper, we extend the Landau approach $^{1,2,25-29}$ to study the key role of the coupling between the non-symmetry-breaking order parameter $q$, describing the electronic instability and a symmetry-breaking instability $\eta$, describing the structural order. The richness of behaviors extracted from the models explains the diversity of spin transition curves reported so far, with non-symmetry-breaking and symmetry-breaking instabilities that may occur simultaneously or sequentially. We discuss how the coupling between parameters may generate sequences of phase transitions, from simple spin crossover to spin transition, with continuous or discontinuous symmetry breaking. The coupling terms enlarge the hysteresis domain of bistability or generate stepwise spin transitions with or without spin-state ordering. 


\section{Spin transition or crossover without symmetry breaking}

The bistable and non-symmetry-breaking nature of molecular spin states can be represented by an Ising variable $\left(q_{i}= \pm 1\right)$ corresponding to one of the two states. This model has been intensively used in the case of spin-crossover molecules: $q_{i}=+1$ corresponds to the high spin (HS) state and $q_{i}=-1$ to the low spin (LS) state. ${ }^{16,30-34}$ What we discuss hereafter is more general than the description of spin transition materials only as the states +1 and -1 may refer to spin state for spin-crossover materials and more generally to one of the bistable molecular states (CT, isomerization...) for other systems.

Different types of thermal spin state conversions occur, depending on the strength of the interactions, of elastic nature, among molecules, from continuous spin-crossover for weak interactions to discontinuous spin transition with thermal hysteresis for strong interactions. The spin-state transition is described through the fraction $\gamma$ of molecules in the high spin state or through the order parameter $q$ :

$$
\gamma=\frac{N_{H S}}{N_{H S}+N_{L S}} \quad \text { and } \quad q=\frac{N_{H S}-N_{L S}}{N_{H S}+N_{L S}}
$$

with $\gamma=\frac{q+1}{2} . N_{H S}$ and $N_{L S}$ denote the number of sites in HS or LS states and $q=+1$ in the full HS state while in the fully LS state $q=-1$. The order parameter $q$ does not break symmetry and the spin transition (ST) is described by expanding the thermodynamic potential of the system in powers of $q$. Since $q$ transforms as the identity representation of the symmetry group, the thermodynamic potential includes all powers of $q$. As discussed in previous works, ${ }^{25,35}$ truncating the potential at fourth order allows for renormalizing $q$ to eliminate the third-order term for simplifying the potential to:

$$
F=A^{\prime}(T) q+\frac{1}{2} B^{\prime} q^{2}+\frac{1}{4} C q^{4}+\lambda_{q} v_{s}\left(\frac{1-q}{2}\right)+\frac{1}{2} C_{S}^{0} v_{s}^{2}
$$

$A^{\prime}(T)$ changes sign with temperature. $C>0$ is required for stability. $\frac{1}{2} C_{S}^{0} v_{s}^{2}$ is the elastic energy related to the total volume strain $v_{S}=\frac{v(T)-v_{H S}}{v_{H S}}$ due to the volume contraction from the HS phase. $\lambda_{q} v_{S}\left(\frac{1-q}{2}\right)$ is the elastic coupling term of $q$ to the volume strain $v_{S}$ that scales as $\left(\frac{1-q}{2}\right)$ to be zero in the high temperature HS phase.

The contribution of the volume strain $v_{s}$, or volume contraction, to the potential is included by finding its equilibrium value that minimizes $(2): v_{S}=-\frac{\lambda_{q}}{C_{S}^{0}}\left(\frac{1-q}{2}\right)$. Substituting $v_{S}$ with this expression in (2) gives the potential: 


$$
F(q)=\left(A^{\prime}+\frac{\lambda_{q}^{2}}{4 C_{s}^{0}}\right) q+\frac{1}{2}\left(B^{\prime}-\frac{\lambda_{q}^{2}}{8 C_{s}^{0}}\right) q^{2}+\frac{1}{4} C q^{4}
$$

Therefore, the elastic coupling shifts the equilibrium line between $q<0$ and $q>0$ and decreases the $q^{2}$ coefficient. We then rewrite the potential as:

$$
F(q, T)=A(T) q+\frac{1}{2} B q^{2}+\frac{1}{4} C q^{4}
$$

Since the goal of the present paper is to discuss the different types of thermal behaviors, we will use potential (4) and consider for pedagogical purpose that $B$ is constant and that $A$ changes linearly with temperature: $A=-a_{0}\left(T-T_{S T}\right)$. We will discuss the temperature dependence of $q$ for different values of $B$. As $A$ and $B$ also depend on pressure, our approach is analogous to crossing the phase transition line of the $(P, T)$ phase diagram not at constant pressure but in an oblique way. The stability condition of $q, \frac{d F}{d q}=0$ and $\frac{d F^{2}}{d q^{2}}>0$, provides the spin transition curve $q(T)$, also given as $\chi(T)$, from predominantly LS $(q<0)$ below $T_{S T}$ to predominantly HS $(q>0)$ above. In this potential, $B>0$ corresponds to a gradual spin state conversion, the so-called spin crossover behavior, with a continuous evolution from $q=1$ at high temperature, $q=0$ at $T=T_{S T}$ and $q=-1$ at low temperature (Figure 1a and $2 \mathrm{a}$ ). $B<0$ promotes cooperativity (Figure $1 \mathrm{~b}$ ) and the resulting spin transition curve $\not(T)$, or $q(T)$, has a the characteristic "S shape" (Figure 2b). Indeed, $F(q)$ exhibits two symmetric stable solutions at $T=T_{S T} ; q= \pm \frac{B}{C}$, while $q=0$ is unstable. The limits of stability of $q>0$ and $q<0$ correspond to temperatures were the first and the second derivatives of the potential are $0: T_{u}=$ $T_{C T}+2 C\left(\frac{-B}{3 C}\right)^{\frac{3}{2}}$ and $T_{d}=T_{C T}-2 C\left(\frac{-B}{3 C}\right)^{\frac{3}{2}}$. The thermal hysteresis, inherent to first order spin transitions, is characterized by its width $\Delta T_{C T}=T_{u}-T_{d} \propto 4 C\left(\frac{-B}{3 C}\right)^{\frac{3}{2}}$.

This model does not provide stable phases at half conversion $(q=0)$ over a broad temperature range, as it is the case for stepwise spin transition discussed in sections IV and V. This phenomenological model is relevant for describing spin conversion. It is clear that the degree of cooperativity, described through the parameter $B$, is the key for explaining spin conversion of spin-crossover type $(B>0)$ or spin-transition type $(B<0)$. Equations $(2)$ and (3) highlight that it is the elastic coupling of $q$ to the volume strain $v_{s}$ that makes the $q^{2}$ coefficient more negative, which is responsible for the appearance of spin transition or spin-crossover. 

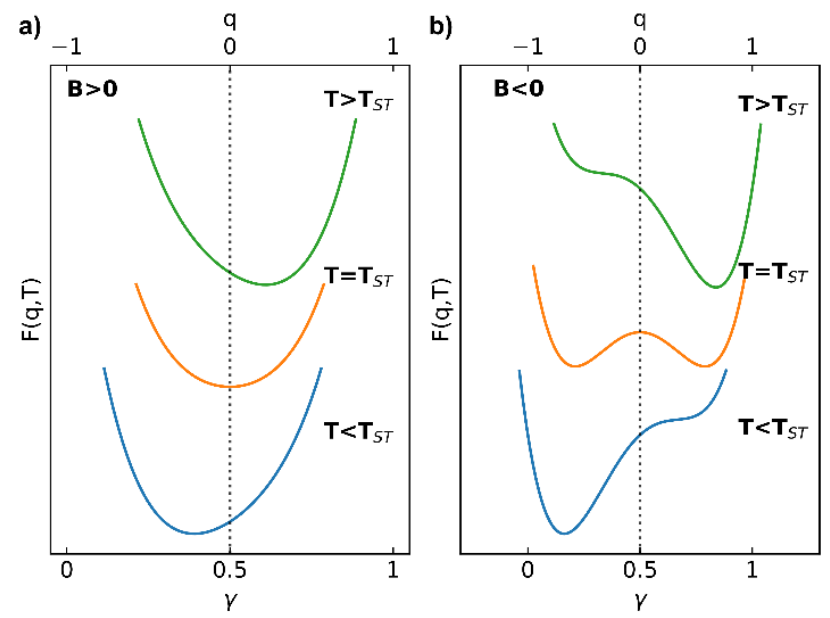

Figure 1 Evolution with temperature of the thermodynamical potential $F(q, T)(4)$ and the equilibrium value of $q$ for $B>0(a)$ and $B<0(b)$.

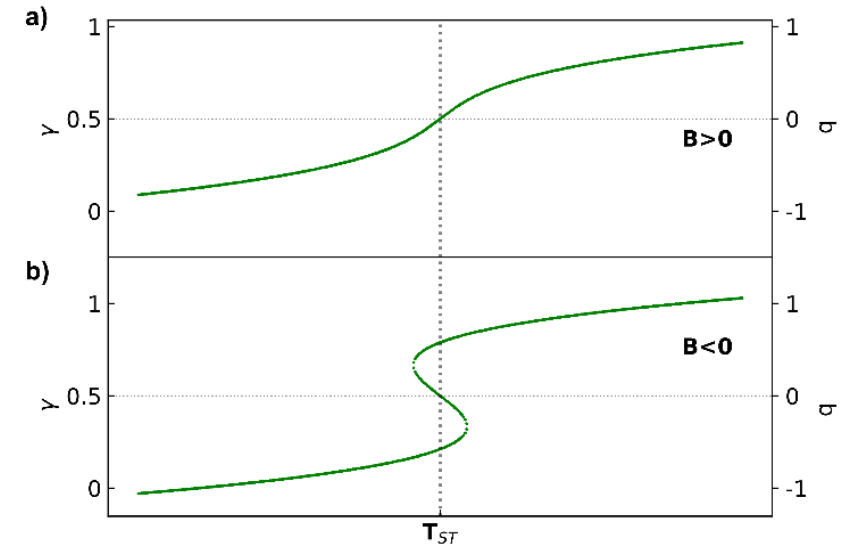

Figure 2 Spin conversion curves $q(T)$ describing a first-order spin transition for $B>0(a)$ and $a$ spin crossover for $B<0(b)$.

The appearance of spin-crossover or spin transition was discussed for the molecular system $\mathrm{Fe}(\mathrm{PM}-\mathrm{BIA})_{2}(\mathrm{NCS})_{2}$, which may crystallize in two polymorphs that show drastically different spin conversions. ${ }^{36-39}$ The orthorhombic polymorph (Pccn) exhibits an abrupt spin transition with a thermal hysteresis (width $\approx 5 \mathrm{~K}$ ), which corresponds to the cooperative case in Figure $2 \mathrm{~b}(B<0$ ), while the monoclinic polymorph $\left(\mathrm{P} 2_{1} / \mathrm{c}\right)$ shows only a gradual spin-crossover type conversion, which corresponds to the crossover case in Figure $2 \mathrm{a}(B>0)$. It was shown that the different intermolecular couplings of both polymorphs play an important role for the degree of cooperativity, ${ }^{40}$ which translates in our model in different coupling strength $\lambda_{q}$ to the volume strain $v_{s}$ in $E q(2)$. 
Various techniques allow monitoring the spin state switching, including Mossbauer spectroscopy, magnetic susceptibility, optical, Raman or X-ray spectroscopies to name a few. ${ }^{41} \mathrm{X}$-ray diffraction can monitor the structural changes at the molecular scale. The average metal-ligand bond length $\langle M-L\rangle$ obtained from structural refinement is the weighted contributions of $\mathrm{HS}$ $\left(<M-L>_{H S}\right)$ and LS $\left(<M-L>_{L S}\right)$ states: $<M-L>(T)=\gamma(T)<M-L>_{H S}+(1-$ $\gamma(T))<M-L>_{H S} \cdot{ }^{28}<M-L>_{H S}$ and $\left.<M-L\right\rangle_{L S}$ are measured in the full HS and LS states and the thermal dependence of the HS fraction, $\gamma(T)$, is experimentally extracted from:

$$
\gamma(T)=\left|\frac{\langle M-L>(T)-<M-L\rangle_{L S}}{\left\langle M-L>_{H S}-<M-L\right\rangle_{L S}}\right|=\frac{q(T)+1}{2}
$$

This simplest model for describing non-symmetry-breaking spin conversion does not allow describing multi-step spin conversion and/or symmetry-breaking phenomena that may couple to the change of spin state. We discuss hereafter how to take into account symmetry breaking and how the coupling of symmetry breaking and spin transition can lead to various types of phase transitions and phase diagrams.

\section{Symmetry breaking phase transition}

Symmetry breaking (SB) may be associated with structural ordering phenomena, including ferroelectric phase transition, ligand, anion/cations motions, or ferroelastic phase transitions ${ }^{8}$ for which the crystal system changes (from cubic to tetragonal, from orthorhombic to monoclinic...). ${ }^{42}$ In many cases, when a symmetry breaking occurs, the low symmetry phase is a subgroup of the high symmetry phase, i.e. some symmetry operators of the high symmetry phase are lost in the low symmetry phase, while the others are preserved. Then, the Landau theory of phase transition can be applied. ${ }^{43}$ For measuring the deviation from the high symmetry phase, the theory uses the amplitude of $\eta$, the symmetry-breaking order parameter (OP), which obeys the transformation properties of the irreducible representation of the symmetry group that is responsible for the symmetry change. The symmetry-breaking OP may have one, two or three dimensions depending of the representation. In the case of ferroelastic phase transitions, the symmetry breaking corresponds to a change from one crystalline system to a lower symmetry one. For example, the cubic-tetragonal ferroelastic distortion is characterized by the difference between the $a$ and $c$ crystalline axis, which are equal in the high symmetry cubic phase: $\eta \propto \frac{(c-a)}{a} .{ }^{25}$ The monoclinictriclinic distortion is characterized by the deviation of lattice angles $\alpha$ and $\gamma$ from $90^{\circ} \ldots$ Symmetry breaking may also occur without change of lattice, due to the relative motions $\Delta \vec{r}$ of atoms from the high symmetry position and $\eta \propto|\Delta r|$. 
The Landau theory describes the evolution of the thermodynamical potential $F$ of the system around the phase transition temperature, through the expansion of $F$ in power series of $\eta$. The orders allowed depend on the representation of the order parameter. Hereafter we discuss the simplest case where $\eta$ is one-dimensional and scalar, which allows two symmetry-equivalent domains $( \pm \eta)$ in the low symmetry phase. Since both domains are equivalent by symmetry, $F(\eta)=F(-\eta)$, only even orders in $\eta$ are allowed. The simplest temperature dependence of $F$ with $\eta$ near the phase transition is given by:

$$
F(\eta, T)=\frac{1}{2} a(T) \eta^{2}+\frac{1}{4} b \eta^{4}
$$

$b>0$ is required for stability. $a(T)=a 0\left(T-T_{S B}\right)$ changes sign at $T_{S B}\left(a_{0}>0\right)$, where the symmetrybreaking phase transition occurs. This potential describes a second-order phase transition between the high and low symmetry phases (Figure 3a), as $\eta$ deviates continuously from 0 below $T_{S B}$ (Figure 4a). We use the same $a_{0}$ in $E q(4)$ and $E q(6)$ in order to reduce the number of parameters in the model.

We may also consider a sixth order term:

$$
F(T)=\frac{1}{2} a(T) \eta^{2}+\frac{1}{4} b \eta^{4}+\frac{1}{6} c \eta^{6}
$$

with $c>0$ for stability. $b>0$ gives the same scenario of continuous phase transition described above. On the contrary, $b<0$ gives rise to a first-order phase transition, for which the high symmetry phase ( $\eta=0)$ is stable down to $T_{S B}$ and the low symmetry phase $(\eta \neq 0)$ is stable up to $T_{1}=\frac{b^{2}}{8 a c}+T_{S B}$ (Figure $3 b$ ). The symmetry-breaking curve $\eta^{2}(T)$ exhibits then a thermal hysteresis where $\eta$ changes discontinuously at $T_{S B}$ and $T_{1}$ (Figure $4 \mathrm{~b}$ ).

A similar first-order symmetry-breaking phase transition was found in the case of the cubictetragonal ferroelastic transition occurring in $\mathrm{RbMnFe}$ charge-transfer systems. In this case, the ferroelastic symmetry-breaking order parameter $\eta$ belongs to a bidimensional representation, ${ }^{25}$ and $F$ includes an $\eta^{3}$ term, limiting symmetry-breaking phase transition to first-order types. Therefore $\eta$ changes discontinuously from the high symmetry phase $(\eta=0)$ to the low symmetry phase $(\eta \neq 0)$ and the thermal evolution of the order parameter $\eta$ is then similar to what is shown in Figure 4a. 

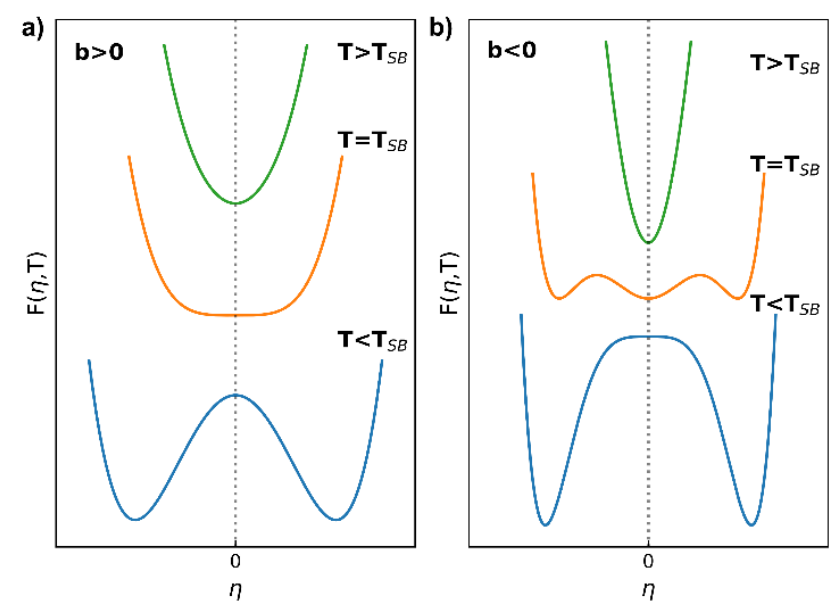

Figure 3 Evolution with temperature of the thermodynamical potentials (7) $F$ and the equilibrium value of $\eta$ for $b>0(a)$ and $b<0(b)$.

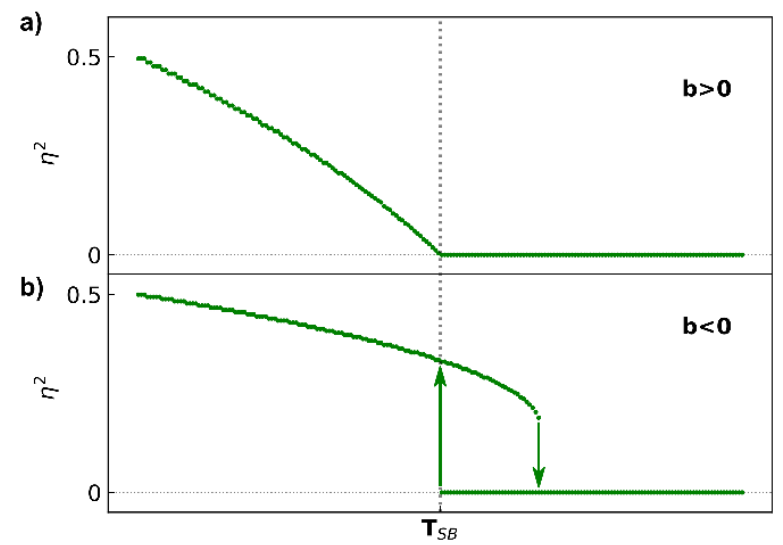

Figure 4 Symmetry breaking curve $\eta^{2}(T)$ extracted from potential (7) describing a second order phase transition for $b>0(a)$ and a first-order phase transition for $b<0(b)$.

The volume change at the molecular scale, due to a redistribution of electrons between more or less bonding states, is an important feature common to many spin-transition materials. In crystals, the average change of electronic state $q$ induces an important volume strain that may reach $10 \%$, as observed in spin transition materials as well as CoFe or MnFe Prussian blue analogues. ${ }^{17,25,44}$ Both the non-symmetry-breaking order parameter $q$ and the symmetry-breaking order parameter $\eta$ can couple to the volume strain, ${ }^{25}$ which results in an effective coupling between $q$ and $\eta^{2}$. Hereafter we consider the total free energy including the contributions of the non-symmetrybreaking order parameter $q$ and the symmetry-breaking order parameter $\eta$, as well as various symmetry-allowed coupling terms. 


\section{Linear-quadratic coupling of spin transition and symmetry-breaking order parameters}

Since $q$ transforms as the identity representation and $\eta$ as a symmetry-breaking representation, the symmetry-allowed coupling term of lowest order is $D q \eta^{2}$. The potential reaches:

$$
F(\eta, q, T)=\frac{1}{2} a \eta^{2}+\frac{1}{4} b \eta^{4}+\frac{1}{6} c \eta^{6}+A q+\frac{1}{2} B q^{2}+\frac{1}{4} C q^{4}+D q \eta^{2}
$$

We studied previously a similar potential, and highlighted the elastic nature of the $D q \eta^{2}$ coupling term. ${ }^{25}$ We use $a_{0}\left(T_{S B}-T_{S T}\right)=-A-a$, to monitor the difference of temperature instability between the symmetry-breaking $\left(T_{S B}\right)$ and the spin $\left(T_{S T}\right)$ transitions. The different phases that may appear in the $\left(T, T_{S B}-T_{S T}\right)$ space, are characterized by the equilibrium values of the OP corresponding to a minimum of the potential in the $(q, \eta)$ space with:

$$
\frac{d F}{d \eta}=0, \frac{d F}{d q}=0, \frac{d F^{2}}{d \eta^{2}}>0, \frac{d F^{2}}{d q^{2}}>0 \text { and } \frac{d F^{2}}{d q d \eta}>0
$$

Here we do not explore the regions of the phase diagram were only the spin transition or only the symmetry breaking transition occurs, as this is already discussed in previous sections. Instead, we focus our attention on the spin transition curve $q(T)$, also scaled in $\chi(T)$, and symmetry breaking curve $\eta(T)$. The two transitions may occur sequentially or simultaneously, depending on $T_{S B}-T_{S T}$ and the coupling strength $D$. The different phases are characterized by their spin state $\left(\gamma>\frac{1}{2}\right.$ for HS, or $\gamma<\frac{1}{2}$ for LS), with high symmetry (hs, $\eta=0$ ) or low symmetry (ls, $\eta \neq 0$ ): HShs, HSls, LShs or LSls. Usually the high temperature phase is High Spin high symmetry (HShs), because high temperature favors both the high entropy HS state and the disordered state (high symmetry). Therefore, we use $D>0$ for stabilizing the low temperature LSls phase $(q<0, \eta \neq 0)$. The relative stability of the different phases depends on $T_{S B}-T_{S T}$, the temperature $T$ and the coupling strength $D$. The order of symmetry-breaking and spin transitions depends on $T_{S B}-T_{S T}$. For the calculations we used $b= \pm 2, B= \pm 2, c=6, C=4, D=2-4$.

Figure 5 shows the phase diagram in the $\left(T_{S B}-T_{S T}, T\right)$ space, when the spin transition $(B<0)$ and the discontinuous symmetry-breaking $(b<0)$, are both associated with thermal hysteresis. Figure 6 shows different sequences of symmetry-breaking and/or spin transitions of continuous or discontinuous nature, calculated for the different combinations ( $b<0, B<0),(b>0, B<0),(b<0, B<0)$ or $(b<0, B>0)$. Each panel shows the curves for $T_{S B}<T_{S T}, T_{S B}=T_{S T}$ and $T_{S B}>T_{S T}$. 


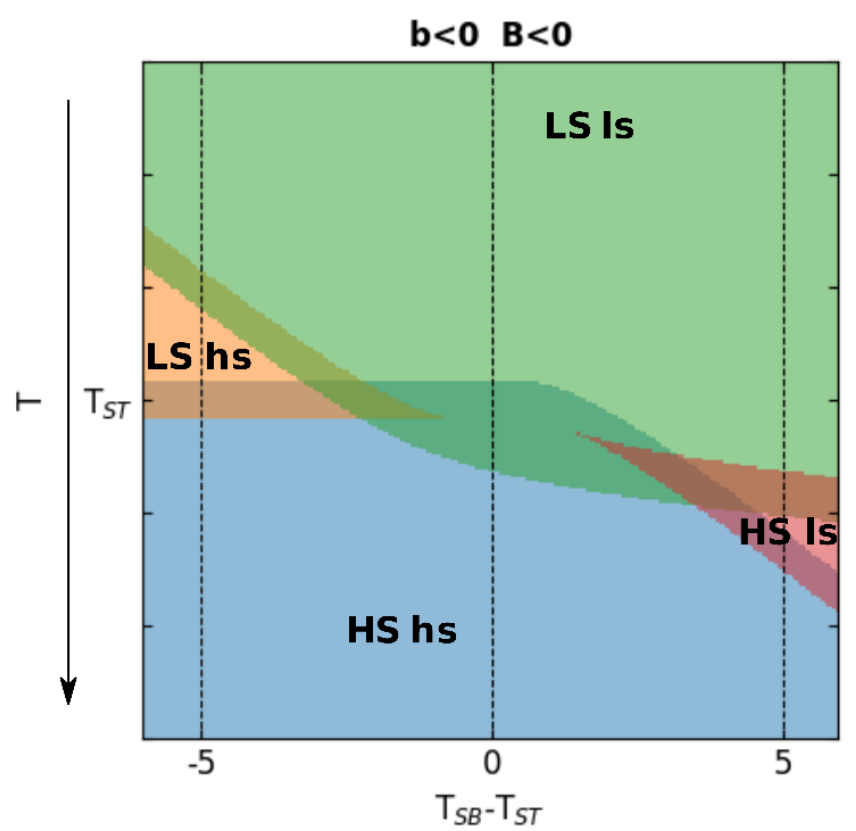

Figure 5 Phase diagrams in the in $\left(T_{S B}-T_{S T}, T\right)$ space exhibiting a spin transition $(B<0)$ and the discontinuous symmetry-breaking $(b<0)$ for $D=2$. The spin transition occurs around $T=T_{S T}$ between low and high spin (LS and HS) states, while the symmetry-breaking temperature occurs at $T_{S B}$ (diagonal) between low and high symmetry ( $l s$ and $\left.h s\right)$ states. The dark thick lines represent the thermal hysteresis and the doted lines are the temperature path shown in Figure 6.

Figure 6a corresponds to discontinuous spin transition (ST) and symmetry breaking (SB) with $(b<0, B<0)$. The results are similar to the ones of the potential including a symmetry-allowed $\eta^{3}$, restricting symmetry-breaking phase transition to first-order types only ${ }^{25}$ When $T_{S T}$ and $T_{S B}$ are different, the symmetry-breaking and spin transitions occur sequentially (top and bottom panels), with intrinsic hysteresis widths. Due to the coupling, the symmetry-breaking phase transition is accompanied by a weak discontinuous change of $q$, and when $T_{S T}$ and $T_{S B}$ are close (middle panel), the symmetry-breaking and spin transition occur simultaneously with a broader hysteresis loop. This case maps several scenarios, including non-symmetry breaking CT-based phase transition in

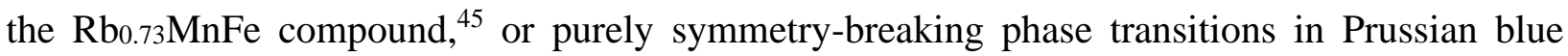
analogues ${ }^{17}$. The broadening of the hysteresis width with the coupling strength, is similar to the broadening observed under chemical pressure, ${ }^{46}$ or the thermal shift of the hysteresis under hydrostatic pressure. ${ }^{47}$ 
a)

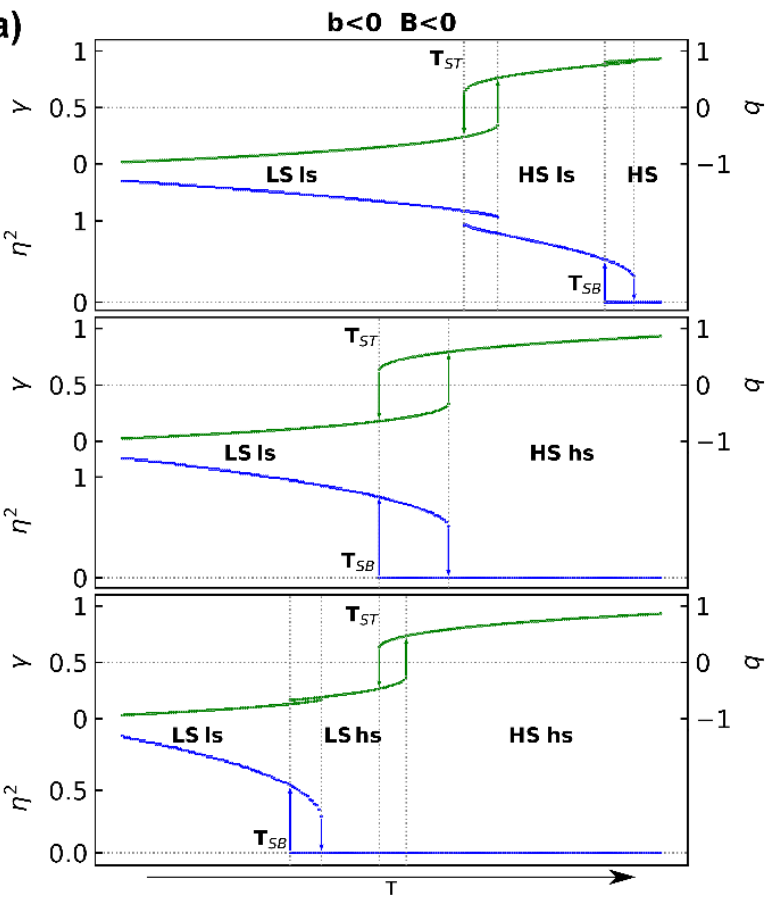

c)

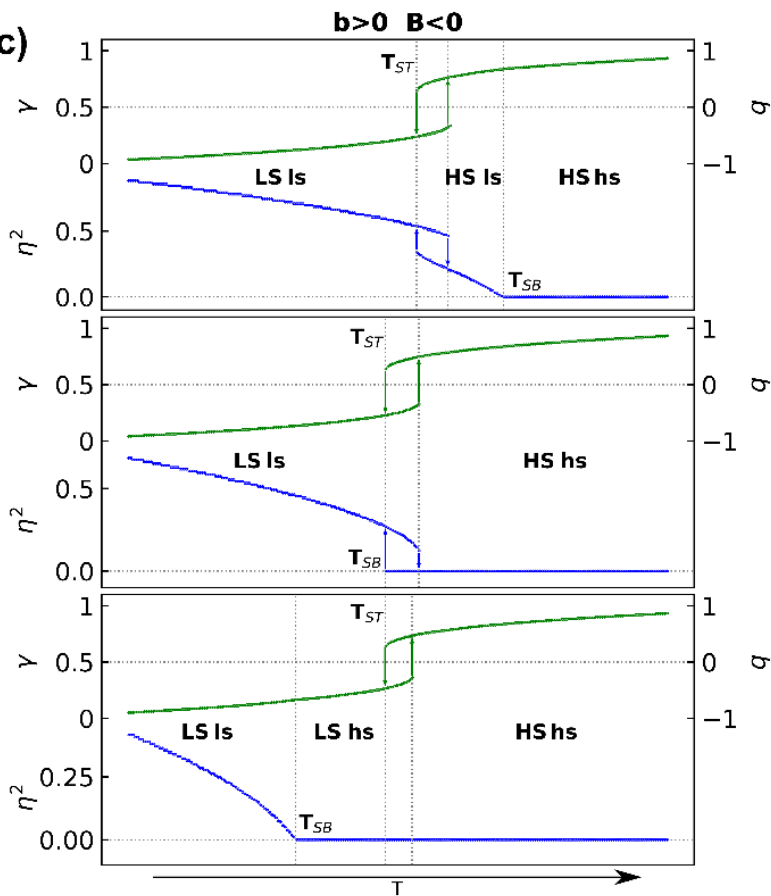

b)

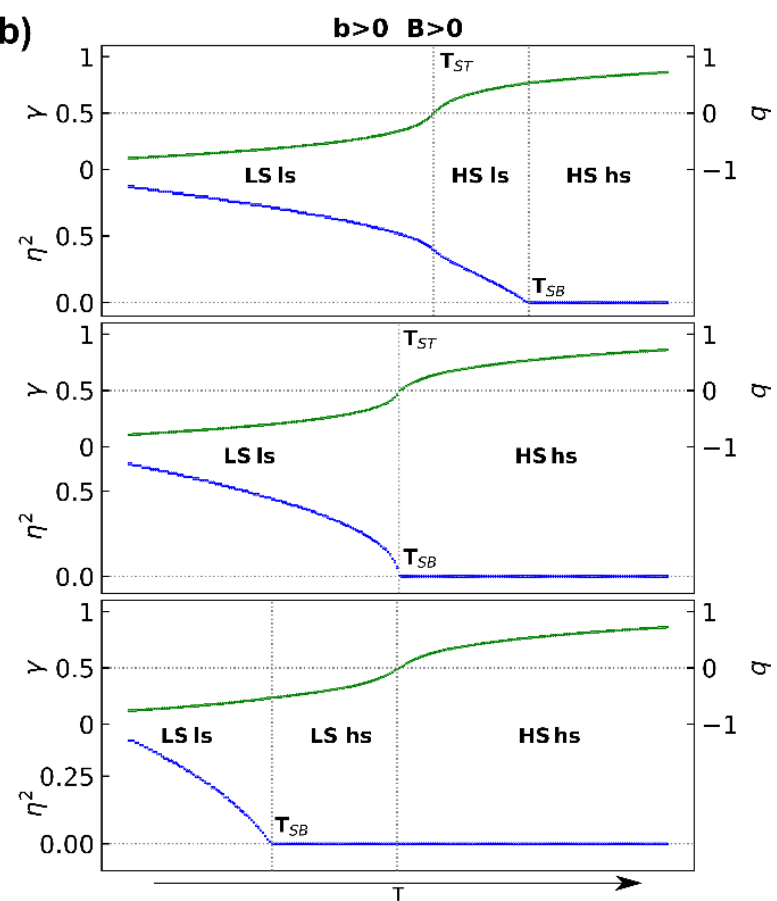

d)

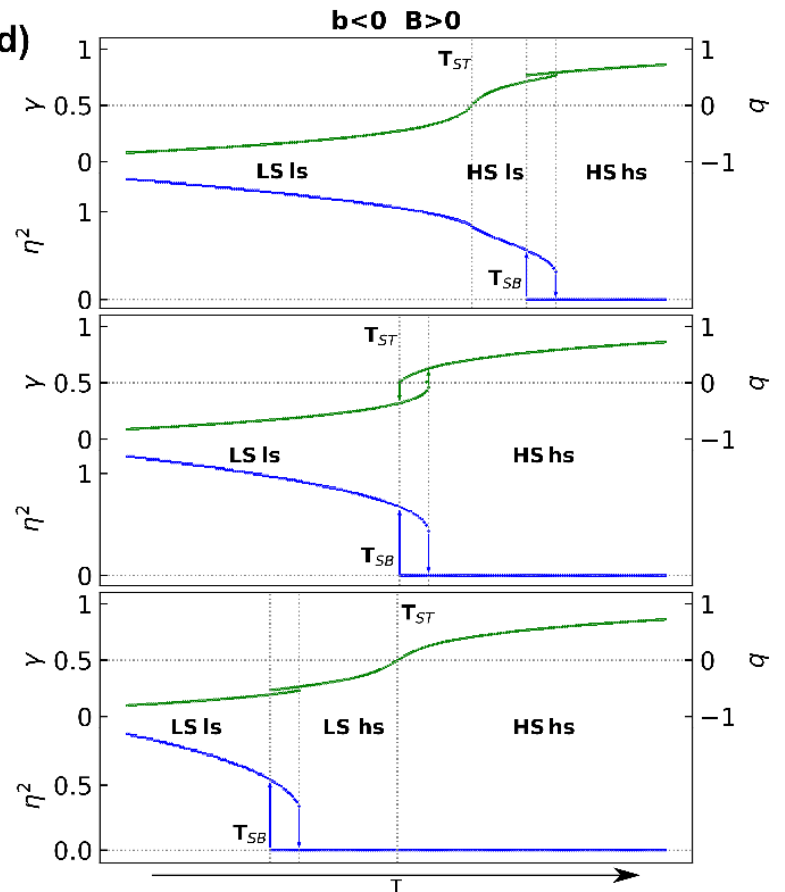

Figure 6 Spin transition curves $\chi(T)$, also scaled in $q(T)$, and symmetry breaking curves $\eta^{2}(T)$. $(a)$, (b), (c) and (d) correspond to the coupling of discontinuous $(b<0, B<0)$ or continuous $(b>0, B>0)$ spin conversion and symmetry breaking. Each panel shows the curves for $T_{S B}<T_{S T}, T_{S B}=T_{S T}$ and $T_{S B}>T_{S T}$. 
Figure $6 \mathrm{~b}$ corresponds to a continuous SB and a discontinuous ST $(b>0, B<0)$. When $T_{S T}<T_{S B}$ the continuous SB occurs at high temperature and the ST below, accompanied by a discontinuous change of $\eta$ due to the coupling. It corresponds to the sequence of phases observed for the $[\mathrm{Mn}(3,5-$ $\operatorname{diBr}_{\left.\left.-\mathrm{sal}_{2}(323)\right)\right] \mathrm{BPh}}$ system $^{48}$ exhibiting a continuous symmetry breaking from $C c$ to $P c$ space groups around $245 \mathrm{~K}$, and a discontinuous spin transition below $90 \mathrm{~K}$. When $T_{S T}$ and $T_{S B}$ are close (middle panel), symmetry breaking and spin transition occur simultaneously due to the coupling and the width of the thermal hysteresis is similar to the one of the spin transition. When $T_{S T}>T_{S B}$ the spin transition occurs at high temperature and a continuous symmetry breaking occurs at low temperature. This last sequence of phase transitions corresponds to the ones observed for the $\left[\mathrm{FeH}_{2} \mathrm{~L}^{2-\mathrm{Me}}\right]\left(\mathrm{ClO}_{4}\right)_{2}$ complex, ${ }^{49}$ exhibiting a cooperative and non-symmetry-breaking spin transition at high temperature followed by a continuous symmetry-breaking at lower temperature. For this system, the symmetry breaking is associated with a cell doubling, resulting from a longrange ordering of bonds on the ligand and motions of the anions. Figure 7 is a schematic representation of the sequence of phases with a spin transition at high temperature and a symmetry breaking at low temperature that may involve cell doubling or ferroelastic distortion.

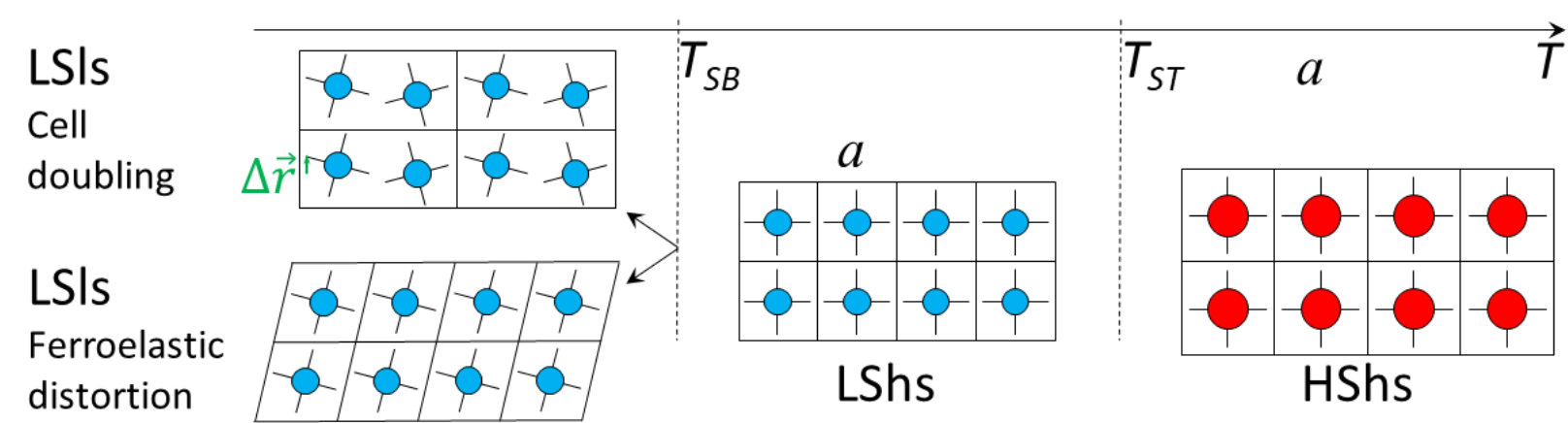

Figure 7 Representation of the sequence of phases with a non-symmetry-breaking spin transition at $T_{S T}$ from HShs to LShs and a symmetry-breaking phase transition at $T_{S B}$ from LShs to LSls. A spin transition occurs with mainly HS molecules above and LS below. The symmetry breaking may have various nature, like cell doubling or ferroelastic distortion, and results in atomic motions $\Delta \vec{r}$, and motions or torsions of ligand, solvent or counter ions.

Figure $6 \mathrm{c}$ corresponds to a continuous symmetry breaking and a continuous spin crossover $(b>0$, $B>0)$ and in this case the effect of the coupling is not pronounced.

Figure $6 \mathrm{~d}$ corresponds to a case, where a discontinuous symmetry breaking phase transition couples to a spin-crossover $(b<0, B>0)$. In the middle panel both phenomena occur simultaneously due to the coupling, as observed for $\left[\mathrm{Co}(\mathrm{dpzca})_{2}\right] .{ }^{50}$ This system exhibits an abrupt and complete 
ST coupled to a tetragonal to monoclinic ferroelastic transition(I4 $1 / \mathrm{a}$ to $\left.\mathrm{P} 2{ }_{1} / \mathrm{c}\right)^{51,52}$ and the potential in equation (8) including only even orders in $\eta$ must be used. A discontinuous symmetry breaking phase transition coupled to a spin-crossover corresponds also to the case of the $\left[\mathrm{Fe}(\mathrm{ptz})_{6}\right]\left(\mathrm{BF}_{4}\right)_{2}$ system. ${ }^{53-58}$ At atmospheric pressure this system exhibits a spin conversion coupled to a first-order ferroelastic phase transition and both phenomena occur simultaneously, ${ }^{54}$ while both phenomena occurs separately under pressure. ${ }^{59,60}$

\section{Non-symmetry-breaking stepwise phase transitions}

Non-symmetry breaking stepwise phase transitions occur in systems for which the crystalline lattice is made of sub-lattices with active molecular sites independent by symmetry. In the case of two sub-lattices, the spin transition of each sub-lattice is monitored by $q_{1}$ and $q_{2}$. We may approximate the total thermodynamical potential $F$ as a sum of the thermodynamical potentials $F_{1}$ and $F_{2}$ of the sub-lattices and the elastic coupling term $F_{c}$ between $q_{1}$ and $q_{2}$ :

$$
F\left(q_{1}, q_{1}\right)=F_{1}+F_{2}+F_{c}=A_{1} q_{1}+\frac{1}{2} B_{1} q_{1}^{2}+\frac{1}{4} C_{1} q_{1}^{4}+A_{2} q_{2}+\frac{1}{2} B_{2} q_{2}^{2}+\frac{1}{4} C_{2} q_{2}^{4}+D_{12} q_{1} q_{2}(10)
$$

When the elastic coupling between the sub lattices is negligible $\left(D_{12}=0\right)$, the global spin conversion is then described through the weighted contribution from each sub-lattice $q=\frac{1}{2}\left(q_{1}+\right.$ $q_{2}$ ). Since the sub-lattices may be associated with different thermodynamical parameters (ligand fields, entropy $\ldots), q_{1}$ may undergo a spin crossover $\left(B_{1}>0\right)$ or a spin transition $\left(B_{1}<0\right)$ around $T_{S T 1}$ and $q_{2}$ may undergo a spin crossover $\left(B_{2}>0\right)$ or a spin transition $\left(B_{2}<0\right)$ around $T_{S T 2}$. The different scenarios shown in Figures $8 \mathrm{a}-\mathrm{c}$ provide more or less pronounced plateaus at half spinconversion $\gamma=\frac{1}{2}$. In this case, the intermediate state at half conversion corresponds to a crystalline structure with molecules mainly in HS state on sub-lattice 1 and LS state on sub-lattice 2.

We discuss now the effect of the elastic coupling $D_{12}$ between two sub-lattices undergoing cooperative spin transitions $\left(B_{1}<0\right.$ and $\left.B_{2}<0\right)$. The coupling may be of anti-ferroelastic nature $\left(D_{12}>0\right)$, which stabilizes sub-lattices in different spin states. This anti-ferroelastic coupling favors therefore the half conversion state at $\gamma=\frac{1}{2}$, for which a single sub-lattice undergoes the spin transition. This state with one sub-lattice mainly HS and one mainly LS is stabilized over a broader temperature range (Figure 8d) compared to the case without coupling (Figure 8c). On the contrary, a ferroelastic coupling $D_{12}<0$ favors the same spin state for both lattices. This ferroelastic coupling destabilizes the half-conversion step and when the coupling is strong enough there is a single step spin transition (Figure 8e), where both sub-lattices switch from LS to HS states simultaneously. 


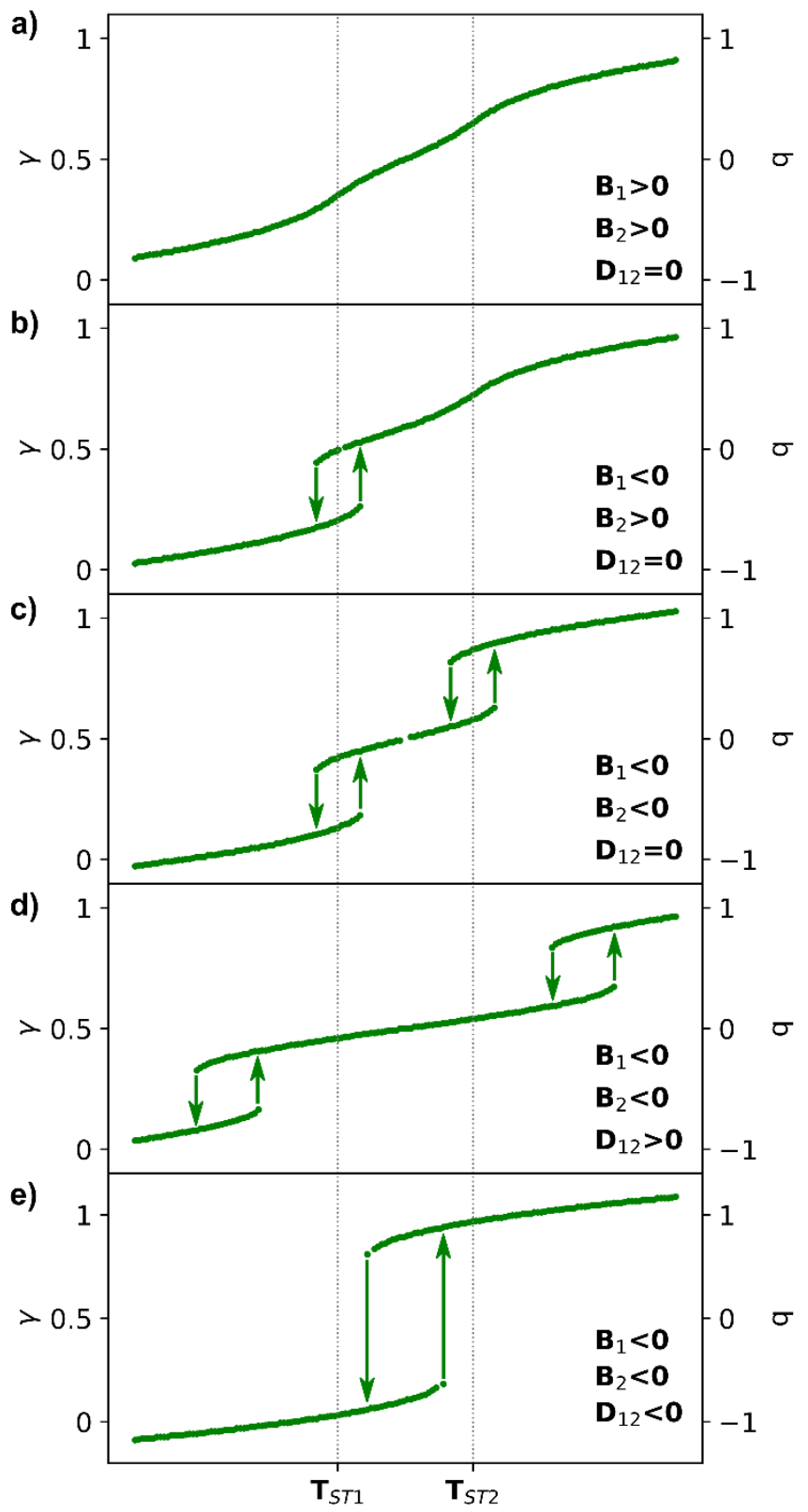

Figure 8 Non-symmetry-breaking stepwise spin transition curves $\chi(T)$ for different potentials (10) including spin crossover and/or spin transition for the two sub-lattices at $T_{S T 1}$ and $T_{S T 2}(a-c)$. The anti-ferroelastic coupling $\left(D_{12}>0\right)$ stabilizes the half conversion step $(d)$, while the ferroelastic coupling $\left(D_{12}>0\right)$ favors a single step spin transition $(e)$.

The compound $\left[\mathrm{Fe}(\mathrm{btr})_{3}\right]\left(\mathrm{ClO}_{4}\right)_{2},{ }^{61}$ exhibits such a LS $\leftrightarrow$ HS spin conversion occurring in two steps: the low-temperature step is very abrupt and occurs with a thermal hysteresis ( $3 \mathrm{~K}$ width), while the high-temperature step is rather gradual. This situation is described in our model with $B_{1}>0$ and $B_{2}<0$ (Figure $8 \mathrm{a}$ ). In this system, there are two different $\mathrm{Fe}^{2+}$ sub-lattices, which interact differently with the anions, and in the intermediate phase the $\mathrm{Fe} 1$ sites remain HS while the Fe2 sites are LS (Figure 9a). Other systems exhibit similar behaviours. ${ }^{62,63}$ 
a) Non-symmetry-breaking stepwise

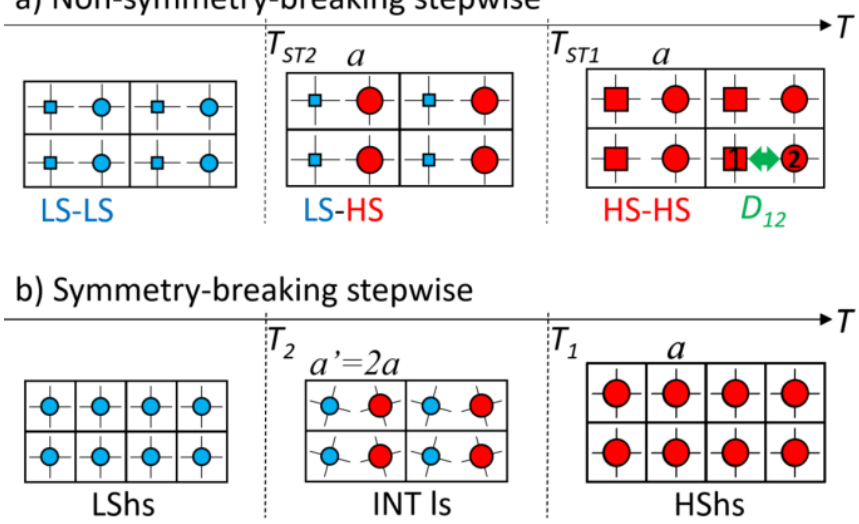

Figure 9 Sequence of phases for stepwise spin transitions. a) Non-symmetry-breaking stepwise spin transition, found in $\left[\mathrm{Fe}\left(\mathrm{btr}_{3}\right)_{3}\left(\mathrm{ClO}_{4}\right)_{2}\right.$ for example, with two sub-lattices (1 square - 2 circle) in the unit cell (rectangle) coupled elastically $\left(D_{12}\right)$. b) Symmetry-breaking stepwise phase transitions, as observed for $\left[\mathrm{Fe}(2-\text { pic })_{3}\right] \mathrm{Cl}_{2} \cdot \mathrm{EtOH}$ or $\left[\mathrm{FeH}_{2} \mathrm{~L}^{2-\mathrm{Me}}\right]\left[\mathrm{PF}_{6}\right]_{2}$ for example, where the symmetry-equivalent sites in the HShs phase are no more equivalent in the INT ls phase, with a doubling of the unit cell for example.

A related but unusual scenario is found in $\left[\mathrm{Fe}(\operatorname{trz}-\mathrm{py})_{2} \mathrm{Pt}(\mathrm{CN})_{4}\right] \cdot 3 \mathrm{H}_{2} \mathrm{O},{ }^{64}$ a two-dimensional Hofmann-like spin transition material made of two symmetrically inequivalent $\mathrm{Fe}^{\mathrm{II}}$ sites. This compound exhibits an incomplete first-order spin transition from a fully high-spin $\left(\mathrm{HS}_{1}-\mathrm{HS}_{2}\right)$ to an intermediate high-spin low-spin $\left(\mathrm{HS}_{1}-\mathrm{LS}_{2}\right)$ phase, without symmetry breaking. The fully lowspin $\left(\mathrm{LS}_{1}-\mathrm{LS}_{2}\right)$ states is not reached at thermal equilibrium but is reached by light irradiation at low temperature. In other words, light excitation driving reverses-LIESST allows accessing to the hidden $\mathrm{LS}_{1}-\mathrm{LS}_{2}$ state, from which the $\mathrm{HS}_{1}-\mathrm{LS}_{2}$ phase is reached upon warming. The double hysteresis (with one hidden at equilibrium) corresponds then to $B_{1}<0$ and $B_{2}<0$.

A similar half spin transition curve was also reported in $\left[\mathrm{Fe}(3 \mathrm{PhOH}-\operatorname{trz})_{2} \mathrm{Pt}(\mathrm{CN})_{4}\right] \cdot 2 \mathrm{H}_{2} \mathrm{O}$ with an hidden hysteresis for the LS-LS phase. ${ }^{65}$ However, the process is different since it is associated with a symmetry change, forming two independent Fe sites in the intermediate phase from a single site in the fully HS and high symmetry phase. We discuss this relation between stepwise transition and symmetry breaking hereafter. 


\section{Stepwise and symmetry breaking spin transition:}

The competition between short-range elastic interaction, favoring alternation of molecules in different spin states with different volume, and long-range elastic interactions due to the global crystalline volume change, favoring molecules in the same spin state, can generate stepwise spin

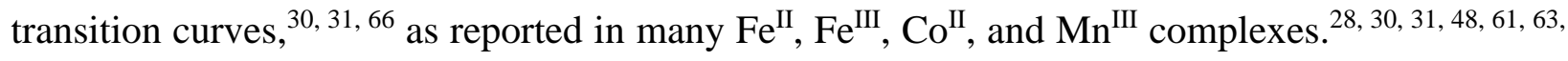
${ }^{67-78}$ Variety of stepwise of processes were studied theoretically. ${ }^{79,80}$ More and more systems are reported to undergo sequences of phases with a fully HS state at high temperature, an intermediate (INT) phase corresponding to a step at half conversion $(q=0)$ and a fully LS phase at low temperature. The INT phase is associated with a long-range and periodic order of molecules in LS and $\mathrm{HS}$ states as observed in $\left[\mathrm{Fe}(2-\mathrm{pic})_{3}\right] \mathrm{Cl}_{2} \cdot \mathrm{EtOH}^{71}$ or $\left[\mathrm{FeH}_{2} \mathrm{~L}^{2-\mathrm{Me}}\right][\mathrm{PF} 6]_{2}$ for example. ${ }^{30}$ However, on the contrary to the case of non-symmetry-breaking stepwise spin transition discussed in section IV with different sub-lattices, in the case of symmetry-breaking stepwise spin transition the mainly HS and LS sites appearing in the INT phase are initially equivalent in the fully HShs phase, and also sometimes in the LS phase as shown in Figure 9b. ${ }^{71,81}$ Therefore, in the INT phase, where the HS fraction is $\gamma \approx \frac{1}{2}$, a symmetry breaking occurs, forming two sub-lattices, one being mainly HS the other mainly LS. The symmetry-breaking order parameter $\eta$ belongs to the active representation of the symmetry group driving the symmetry change. Its amplitude describes the amplitude of the difference between the molecular sites forming the sub-lattices, including the different HS fraction: $\eta=\frac{1}{2}\left(q_{1}-q_{2}\right)$. The symmetry change can also involve atomic motions, ligand or lattice torsion and rotation... As it is the case for non-symmetry-breaking spin transition, the HShs phase is characterized by $(0<q \leq 1, \eta=0)$ and the LShs phase is characterized by $(-1 \leq q<0, \eta=0)$. In the INT phase, if $q=0$ and the sub-lattice 1 is fully HS and 2 is fully LS $(\eta=1)$ the order is complete. The other domain, or the antiphase depending on the nature of the symmetry-breaking, corresponds to sub-lattice 1 fully LS and 2 fully HS ( $\eta=-1)$. These two domains can equally form, and in most cases the order is partial in the symmetry-broken INT phase $(0<|\eta|<1)$. Here again, a phase transition where spin transition and symmetry breaking occur simultaneously requires coupling between $q$ and $\eta$. In addition to the symmetry-allowed coupling term $D q \eta^{2}$ discussed above, it is the bi-quadratic coupling term $E q^{2} \eta^{2}$, with $E>0$, that stabilizes the half conversion step, by minimizing the thermodynamical potential around $q=0$ for $\eta \neq 0$. For limiting the number of terms in the potential, we neglect the $D q \eta^{2}$ term, which shifts the stability region of the INT phase as explained by Chernyshov, ${ }^{35}$ and we use the most simple potential:

$$
F(\eta, q)=\frac{1}{2} a \eta^{2}+\frac{1}{4} b \eta^{4}+A q+\frac{1}{2} B q^{2}+\frac{1}{4} C q^{4}+E q^{2} \eta^{2}
$$


Here again the stability condition is found with: $\frac{d F}{d \eta}=0=\left(a+2 E q^{2}+b \eta^{2}\right) \eta=0$.

$\eta=0$ is a trivial solution and corresponds to the non-symmetry-breaking spin transition case.

$\eta \neq 0$ is found for $\eta^{2}=-\frac{a+2 E q^{2}}{b}$, with a stability condition $\frac{d F^{2}}{d \eta^{2}}=a+3 b \eta^{2}+2 E q^{2}>0$.

Substituting $\eta^{2}$ leads to $a+2 E q^{2}<0$, which can also be written $T-T_{S B}<2 \frac{E}{a_{0}} q^{2}$. The low symmetry INT phase with HS-LS order and $\eta \neq 0$ is then stabilized in a temperature range $\left[T_{S B 2}-T_{S B 1}\right]$ around $q=0$. Figure 10 shows the spin transition curve $q(T)$ and the symmetry breaking curve $\eta^{2}(T)$ corresponding to cooperative symmetry-breaking stepwise transitions, with thermal hysteresis.

In many cases reported so far, the symmetry breaking is associated with a change of translation symmetry (cell doubling, change of lattice type or crystalline system) or the loss of nonsymmorphic symmetry operators (screw axis or glide planes), making molecular sites inequivalent in the crystal. The appearance of new Bragg peaks related to such symmetry-breaking is then often a direct characterization of the symmetry change, and the intensity of the Bragg peaks is proportional to $\eta^{2} \cdot{ }^{30}$ A more direct way to probe the amplitude of the symmetry breaking due to the ordering of HS and LS molecules on different sub-lattices, is to measure by diffraction techniques the difference of the average metal-ligand bond lengths $\langle\mathrm{M}-\mathrm{L}\rangle$ between sub-lattices 1 and $2 .{ }^{28} \eta$ scales with the difference between the sites normalized to the difference between fully HS and fully LS states:

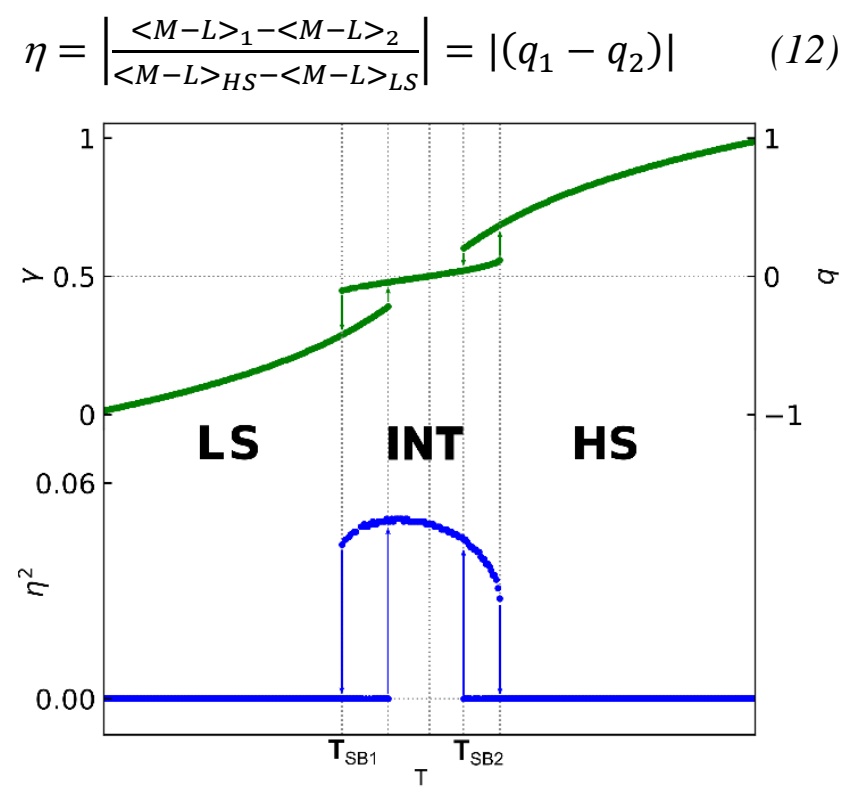

Figure 10 Symmetry-breaking stepwise spin transition curves $\chi(T)$ and $\eta^{2}(T)$ for potential (11). 
Here again, the order is complete when one sub-lattice is fully HS and the other one fully LS, i.e. when the bond lengths $\langle\mathrm{M}-\mathrm{L}\rangle$ of one sub-lattice site corresponds to the LS state and the one of the other sub-lattice to the HS state. For partial order the symmetry is broken $(0<|\eta|<1)$ and the bond lengths are intermediate between the fully HS and LS states. When the phase transition is continuous, the difference between the sites is weak and reaches zero at the phase transition, and the signal related to symmetry breaking is therefore more difficult to observe. ${ }^{30}$

As shown in Figs. 10 and 11, $\gamma$ may evolve with temperature on approaching the INT phase, above $T_{S B 1}$ or below $T_{S B 2}$, as well as in the INT phase. The discontinuous change of $\eta$ at $T_{S B 1}$ and $T_{S B 2}$ is accompanied by a discontinuous change of $\gamma$ due to the coupling. The high temperature step is less discontinuous than the low temperature step due to entropy. Various scenarios may appear with i) two strongly discontinuous phase transitions, ii) a half spin crossover at high temperature with a continuous symmetry change and a discontinuous phase transition at low temperature iii) two continuous symmetry-breaking phase transitions with a continuous change of $q$. Chernyshov described a similar case, with two discontinuous symmetry-breaking phase transitions, without thermal hysteresis. ${ }^{35}$ Since the HS fraction $\gamma$ differs on the sub-lattices, the long-range HS-LS ordered structure forms the so-called spin-state concentration waves (SSCW), ${ }^{27,28,82}$ which is a meaningful way to describe the spin state order. As temperature decreases, the average HS fraction $\gamma$ decreases from 1 to 0 ( $q$ evolves from 1 to -1$)$. In the symmetry-broken INT phase there is a spatial modulation of the HS fraction over the different sites around the average value $\gamma$ (related to an average $q$ ) and the amplitude of the SSCW is related to the symmetry-breaking order parameter $\eta$. The symmetry breaking due to HS-LS ordering relaxes structural constrains and may therefore be accompanied by rotation, torsion or motion of the molecules, counter-ions or solvent. 

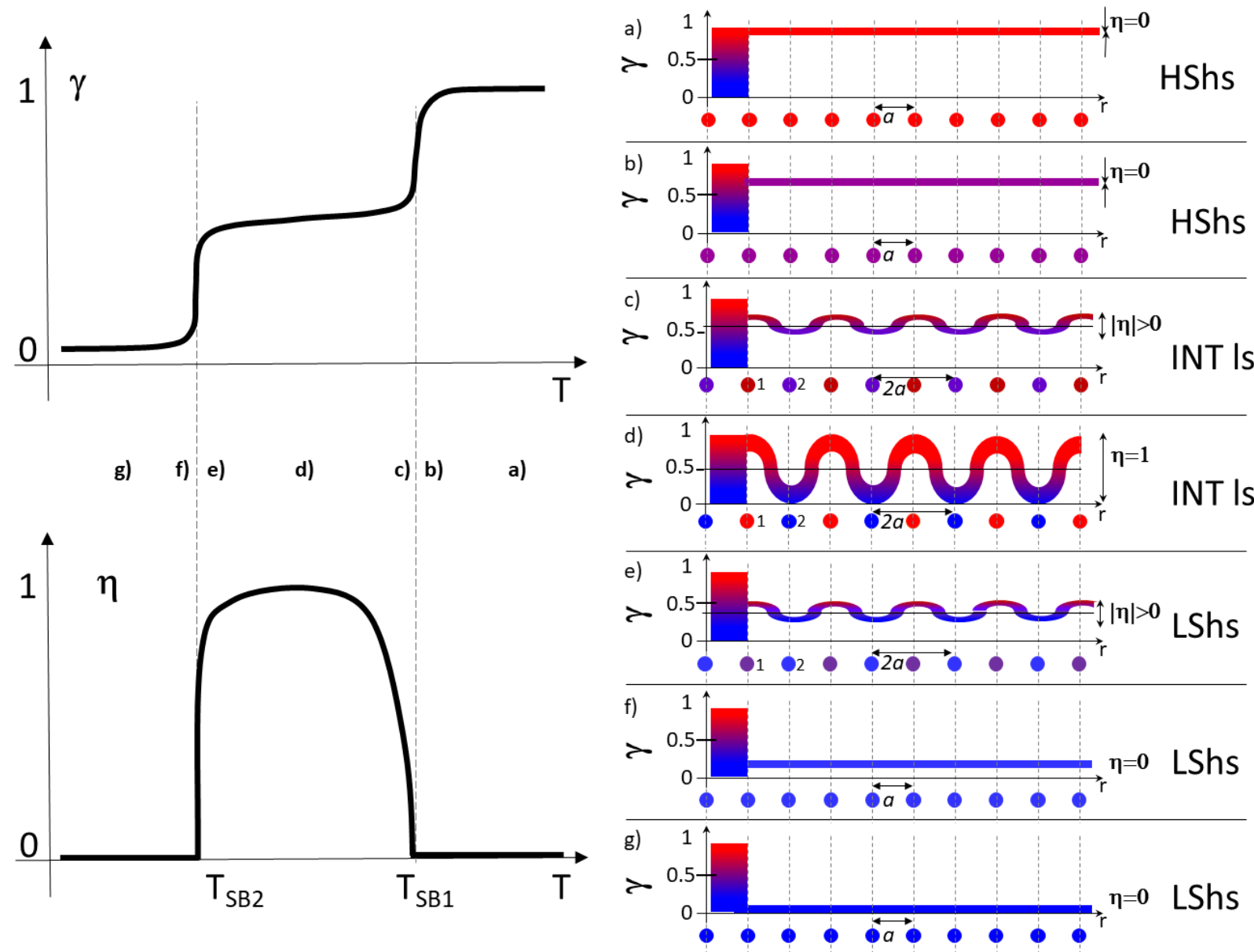

Figure 11 Schematic representation of the average HS fraction $\gamma$ and symmetry-breaking order $\eta$ at different temperatures (a-g) during the symmetry-breaking stepwise phase transition.

\section{Stepwise and reconstructive spin transition:}

The $\left[\mathrm{Fe}(3 \mathrm{PhOH}-\mathrm{trz})_{2} \mathrm{Pt}(\mathrm{CN})_{4}\right] \cdot 2 \mathrm{H}_{2} \mathrm{O}$ system exhibits an unusual behavior associated with a half spin conversion from the fully HS phase $(P n)$ to a long-range ordered HS-LS phase $(P c){ }^{65}$ However, there is no group-subgroup relationship between the two space groups, as the HS and HS-LS phases share the same $a, b, c$ lattice. The symmetry change comes from the fact that the four sites in the unit cell split in two sub-lattices (Figure 12). In the Pn HS phase, for each sublattice one site is equivalent to another one by the $\mathrm{n}$ glide plane. Both sub-lattices are HS. In the $P c$ HS-LS phase, the sites are no more equivalent within the same sub-lattice of the HS phase, one site being mainly HS and the other one mainly LS, as the $n$ plane is lost. However, the two HS sites from the different sub-lattices of the Pn phase, initially inequivalent in the HS phase, become equivalent by the $c$ glide plane in the $P c$ phase. Therefore, the two LS sites initially inequivalent in the HS phase also become equivalent by the $c$ glide plane. Such a structural reorganization is reconstructive and the Landau theory does not apply. Indeed, in this case, there is no symmetry 
deviation from a high symmetry phase, there are just two different phases with different symmetries. This HS-LS phase is stable down to $10 \mathrm{~K}$ or less. Reverse-LIESST allows reaching a fully LS state, which as the same $P c$ space group of the [HS-LS] phase, but both Fe sites are now LS. This reverse-LIESST, without symmetry change, from a phase with HS and LS sub-lattices, is then analogous to the reverse-LIESST from the HS-LS phase found in [Fe(trzpy) $\left.{ }_{2} \mathrm{Pt}(\mathrm{CN})_{4}\right] \cdot 3 \mathrm{H}_{2} \mathrm{O} .{ }^{64}$

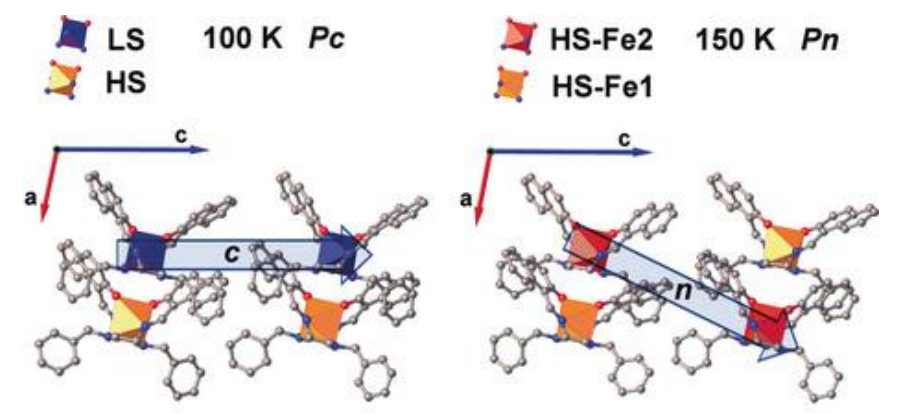

Figure 12 The reconstructive phase transition in [ Fe(3PhOH-trz $\left.)_{2} \mathrm{Pt}(\mathrm{CN})_{4}\right] \cdot 2 \mathrm{H}_{2} \mathrm{O}$. In the HS phase $(150 \mathrm{~K})$ there are two $\mathrm{Fe} 1$ and $\mathrm{Fe} 2$ sub-lattices and the Fe centers of each sub lattice are equivalent by the $n$ glide plane. Different sub lattices form in the HS-LS phase (100 K) one LS and HS and the Fe centers of each sub lattice are equivalent by the $c$ glide plane. Reproduced with permission from Angew. Chem. Int. Ed. 58, 11811 (2019). Copyright 2019 John Wiley and Sons.

The case of multi-step phase transition is also another example of reconstructive phase transitions, as each step corresponds to a spin state concentration wave. Each SSCW is described by the modulation of the HS population of the molecular sites in position $r$ :

$$
\gamma(\boldsymbol{r})=\gamma+\frac{\eta}{2} \cos (\boldsymbol{Q} \cdot \boldsymbol{r})
$$

$\boldsymbol{Q}_{\boldsymbol{R}}=\frac{2 \pi}{R}$ and $R$ as the periodicity of the SSCW, $\eta$ is the amplitude of the wave (order) and $\gamma$ is the average HS fraction. In the case of Figure $11, \boldsymbol{R}=2 \boldsymbol{r}$, which corresponds to a cell doubling along the crystalline axis $\boldsymbol{a}$ for example. There are more and more examples of systems undergoing multi-step SCO, for which the different steps correspond to different SSCW. Figure 13 presents the analogy with the Devil's staircase concept, ${ }^{27,30,83}$ allowing for a simple, but relevant, description of the different spin state ordering on the steps. As observed experimentally ${ }^{27,28,61,84-}$ 86 different spatial modulation of HS (+) and LS (-) states may appear on the steps, with a periodicity $\boldsymbol{R}=m \boldsymbol{r}$ increased with respect to the original lattice periodicity $\boldsymbol{r}$. The HS fraction is then locked onto a rational number $\gamma=\frac{n}{m}$ with n HS sites over $\mathrm{m}$ sites. Theoretically there is an 
infinity of steps between any two steps, as there is an infinity of rational numbers $\frac{n}{m}$ lying in between any two rational numbers. That's the reason why such a stepped sequence of phases is called the Devil's staircase. Cruddas and Powell used a simple model of elastic interactions and identify thirty six different spin-state ordered phases. ${ }^{87}$ Boukheddaden used an elastic model, taking into account both volume change upon spin transition and elastic frustration to explain the existence of organized spatial modulations of HS state inside the plateau of two-step spin transitions. ${ }^{31}$ The stabilization of the steps is balanced by the competition between short-range and long-range interactions. ${ }^{30}$

The $\left[\mathrm{Fe}^{\mathrm{II}}(\right.$ bipydz $\left.)\left(\mathrm{Au}^{\mathrm{I}}(\mathrm{CN})_{2}\right)_{2}\right] \cdot 4(\mathrm{EtOH})$ systems illustrates perfectly this concept of multi-step SSCW. This 3D Hofmann-like material exhibits an hysteretic four-step spin conversion, between the high symmetry HS phase $(\gamma=1)$ and LS phase $(\gamma=0)$ with three intermediate phases corresponding to the steps $\gamma=\frac{2}{3}, \gamma=\frac{1}{2}$ and $\gamma=\frac{1}{3}$, with a long-range periodic ordering of spin states. ${ }^{86}$ This situation corresponds to the steps shown in red in Figure 13. However, each step has its own lattice periodicity $\boldsymbol{R}$. Compared to the HS phase with periodicity $\boldsymbol{r}$, the periodicity is $3 \boldsymbol{r}$ for the steps at $\gamma=\frac{2}{3}(++-$ sequence $)$ and $\gamma=\frac{1}{3}(+--$ sequence $)$, while on the step at $\gamma=\frac{1}{2}$ the periodicity is $2 \boldsymbol{r}(+-+-$ sequence). From the point of view of translation symmetry, the phase formed by the SSCW at $\gamma=\frac{2}{3}, \gamma=\frac{1}{2}$ and $\gamma=\frac{1}{3}$ are sub-groups of the HS phase. However the translation symmetry $3 \boldsymbol{r}$ allowed on the steps $\gamma=\frac{2}{3}$ and $\gamma=\frac{1}{3}$ is forbidden for $\gamma=\frac{1}{2}$, and symmetrycally the translation symmetry $2 \boldsymbol{r}$ allowed on the step $\gamma=\frac{1}{2}$ is forbidden for $\gamma=\frac{2}{3}$ and $\gamma=\frac{1}{3}$. Therefore there is no group-subgroup relationship between the different phases on the steps and the phase transition between each step are reconstructive. Consequently, in this case the phase transition between the steps are limited to first-order, except for the first and last steps with the high symmetry phases. However, the concept of order parameter also applies for measuring the deviation from the HShs phase. ${ }^{27,30}$ The description of aperiodic SSCW, with a spatial modulation of the HS fraction incommensurate with the lattice periodicity, remains more challenging, even though the concept of order parameter also applies to the super space approach. ${ }^{27,88}$ 


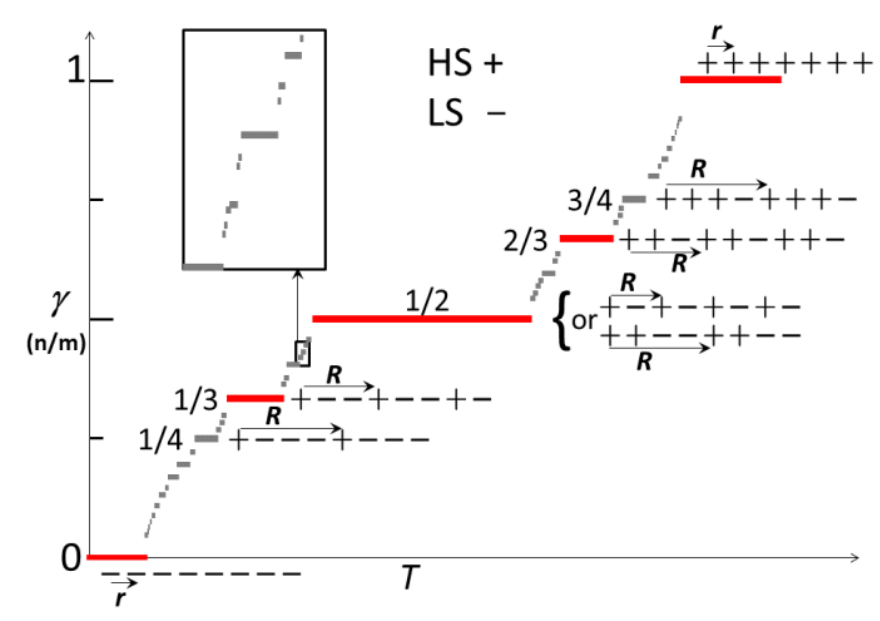

Figure 13 Devil's staircase for SCO materials, the steps with T correspond to fractions $\gamma=\frac{n}{m}$ with long-range ordering of $L S(-)$ and $H S(+)$ sites. The modulation vector $\boldsymbol{R}=n \boldsymbol{r}$ and there are $n H S$ sites among $m$ sites. Structure of the simplest (thick segments) steps are represented. Reproduced with permission from Angew. Chem. Int. Ed. 55, 8675 (2016). Copyright 2016 John Wiley and Sons.

\section{Conclusion}

The Landau theory, with proper identification and use of symmetry-breaking and non-symmetrybreaking order parameters is very useful to describe phase transitions in multi-stable materials. Indeed, non-symmetry-breaking instabilities related to the OP $q$, monitoring change of spin state or $\mathrm{CT}$, and a symmetry-breaking instability related to an $\mathrm{OP} \eta$, monitoring ferroelastic, ferromagnetic, ferroelastic or structural orders, may occur simultaneously, or sequentially. The phenomenological approach presented here, discussing the coupling between the order parameters, provides the necessary formalism for describing and disentangling spin transition and symmetry breaking. The diverse spin transition curves $\chi(T)$, obtained for the different types of potentials, map most of the scenarios found in spin-transition materials. These include spin-crossover and spin transition, coupled or not to continuous or discontinuous symmetry-breaking, with or without intermediate step.

We can also found in the literature situations that are more complex than the ones listed in the different sections above. Some of them correspond to a mixing of the scenarios discussed in this paper. For example, in the case of the $\left[\mathrm{FeH}_{2} \mathrm{~L}^{2-\mathrm{Me}}\right]\left[\mathrm{PF}_{6}\right]_{2}$ and $\left[\mathrm{FeH}_{2} \mathrm{~L}^{2-\mathrm{Me}}\right]\left[\mathrm{AsF}_{6}\right]_{2}$ systems, ${ }^{67,68}$ an INT phase associated with HS-LS order forms on cooling from the HShs phase, which correspond to the stepwise and symmetry breaking spin transition scenario. However, the LS phase is a low 
symmetry one (LSls) with a symmetry breaking different from the one occurring on the step. The HShs and LSls phases correspond then to the linear-quadratic coupling scenario between spin transition and symmetry-breaking order parameters. The potential to be used to map these mixed scenarios is more complex, since different symmetry-breaking order parameters have to be used: one for describing the HS-LS order and one for the structural distortion in the LSls phase. The $\left[\mathrm{Fe}(\mathrm{salpm})_{2}\right] \mathrm{ClO}_{4} \cdot 0.5 \mathrm{EtOH}$ complex corresponds to another mixed case. It is made of two sublattices in the HShs phase. On cooling, one sub-lattice remains HS and the other one undergoes a half spin conversion with HS-LS symmetry-breaking order. At lower temperature, both sublattices switch to the LS state. This scenario is a mixture of a stepwise symmetry breaking spin transition for one sub-lattice and a spin-transition on the other. Here again, the potential required to map this mixed scenario requires including a coupling between the sub-lattices to explain their simultaneous spin transition at lower temperature.

The phenomenological approach discussed in this paper allows for a rigorous description and understanding of spin transition materials, by explaining how to disentangle the non-symmetrybreaking (spin state, charge-transfer...) and the symmetry-breaking (ferromagnetic, ferroelectric, ferroelastic, structural order...) instabilities. The elastic coupling between both types of instabilities can enhance the hysteresis width, drive simultaneous spin transition and symmetrybreaking and generate stepwise spin transitions. The coupling between symmetry-breaking and non-symmetry-breaking instabilities is also an important route for enhancing the (multi-) functionalities. For example, charge transfer can enhance the electric dipole, which, combined to ferroelectric order, enhances the electric polarization. Spin transition and charge transfer can enhance the magnetic dipole, which combined to ferromagnetic order enhances the magnetization. Mixing symmetry-breaking and non-symmetry-breaking instabilities is therefore an important route for understanding, controlling and developing multi-functional materials.

\section{Acknowledgement}

The authors gratefully acknowledge Agence Nationale de la Recherche for financial support undergrant ANR-16-CE30-0018, ANR-19-CE30-0004 and University Rennes 1 for funding. We thank C. Ecolivet, H. Cailleau and S. Miyashita for scientific discussions.

\section{Availability of data}

The data that support the findings of this study are available from the corresponding author upon reasonable request. 


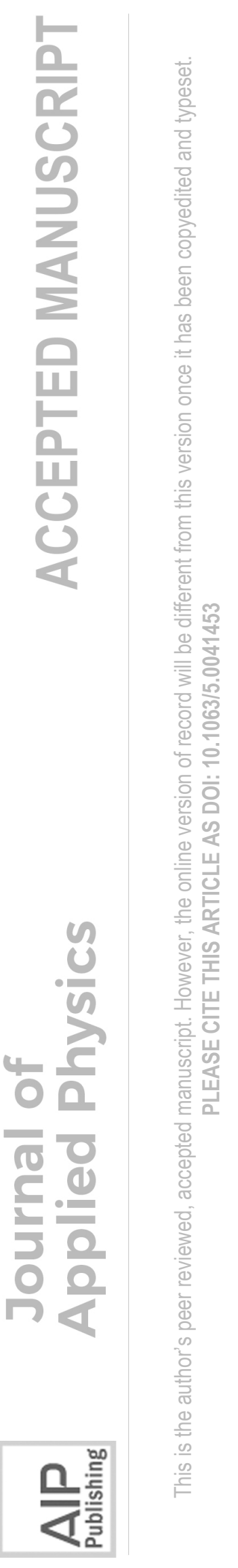




\section{References}

1. M. A. Halcrow, Spin-crossover materials : properties and applications. (Wiley, 2013).

2. P. Gutlich, A. Hauser and H. Spiering, Angewandte Chemie-International Edition in English 33 (20), 2024-2054 (1994).

3. A. Bousseksou, G. Molnar, L. Salmon and W. Nicolazzi, Chemical Society Reviews 40 (6), 3313-3335 (2011).

4. H. Tokoro and S. Ohkoshi, Bulletin of the Chemical Society of Japan 88 (2), 227-239 (2015).

5. Y. S. Meng, O. Sato and T. Liu, Angew Chem Int Ed Engl 57 (38), 12216-12226 (2018).

6. E. Pardo, C. Train, H. Liu, L.-M. Chamoreau, B. Dkhil, K. Boubekeur, F. Lloret, K. Nakatani, H. Tokoro, S. Ohkoshi and M. Verdaguer, Angewandte Chemie 124 (33), 8481-8485 (2012).

7. D. Aguila, Y. Prado, E. S. Koumousi, C. Mathoniere and R. Clerac, Chemical Society Reviews 45 (1), 203-224 (2016).

8. E. Collet, M. H. Lemee-Cailleau, M. Buron-Le Cointe, H. Cailleau, M. Wulff, T. Luty, S. Y. Koshihara, M. Meyer, L. Toupet, P. Rabiller and S. Techert, Science 300 (5619), 612-615 (2003).

9. S. Koshihara, Y. Takahashi, H. Sakai, Y. Tokura and T. Luty, The Journal of Physical Chemistry B $\mathbf{1 0 3}$ (14), 2592-2600 (1999).

10. Y. Kawakami, S. Iwai, T. Fukatsu, M. Miura, N. Yoneyama, T. Sasaki and N. Kobayashi, Phys Rev Lett 103 (6), 066403 (2009).

11. T. Ishikawa, S. A. Hayes, S. Keskin, G. Corthey, M. Hada, K. Pichugin, A. Marx, J. Hirscht, K. Shionuma, K. Onda, Y. Okimoto, S.-y. Koshihara, T. Yamamoto, H. Cui, M. Nomura, Y. Oshima, M. Abdel-Jawad, R. Kato and R. J. D. Miller, Science 350 (6267), 1501-1505 (2015).

12. M. Hojorat, H. Al Sabea, L. Norel, K. Bernot, T. Roisnel, F. Gendron, B. L. Guennic, E. Trzop, E. Collet, J. R. Long and S. Rigaut, Journal of the American Chemical Society 142 (2), 931-936 (2020).

13. M. Irie, T. Fukaminato, K. Matsuda and S. Kobatake, Chemical Reviews 114 (24), 12174-12277 (2014).

14. O. Sato, Nat Chem 8 (7), 644-656 (2016).

15. D. B. McWhan, A. Menth, J. P. Remeika, W. F. Brinkman and T. M. Rice, Physical Review B 7 (5), 1920-1931 (1973).

16. H. Spiering, K. Boukheddaden, J. Linares and F. Varret, Physical Review B 70, 184106 (2004).

17. A. Bleuzen, J.-D. Cafun, A. Bachschmidt, M. Verdaguer, P. Münsch, F. Baudelet and J.-P. Itié, The Journal of Physical Chemistry C 112 (45), 17709-17715 (2008).

18. N. Shimamoto, S. Ohkoshi, O. Sato and K. Hashimoto, Inorganic Chemistry 41 (4), 678-684 (2002).

19. N. Ozaki, H. Tokoro, Y. Hamada, A. Namai, T. Matsuda, S. Kaneko and S. Ohkoshi, Advanced Functional Materials 22 (10), 2089-2093 (2012).

20. V. Escax, A. Bleuzen, J. P. Itié, P. Munsch, F. Varret and M. Verdaguer, The Journal of Physical Chemistry B 107 (20), 4763-4767 (2003).

21. V. Escax, A. Bleuzen, C. Cartier dit Moulin, F. Villain, A. Goujon, F. Varret and M. Verdaguer, Journal of the American Chemical Society 123 (50), 12536-12543 (2001).

22. A. C. Felts, M. J. Andrus, E. S. Knowles, P. A. Quintero, A. R. Ahir, O. N. Risset, C. H. Li, I. Maurin, G. J. Halder, K. A. Abboud, M. W. Meisel and D. R. Talham, The Journal of Physical Chemistry C 120 (10), 5420-5429 (2016).

23. H. Tokoro, T. Matsuda, K. Hashimoto and S. Ohkoshi, Journal of Applied Physics 97 (10), 10M508 (2005).

24. S. Ohkoshi, Y. Tsunobuchi, T. Matsuda, K. Hashimoto, A. Namai, F. Hakoe and H. Tokoro, Nat Chem 2 (7), 539-545 (2010).

25. G. Azzolina, R. Bertoni, C. Ecolivet, H. Tokoro, S. Ohkoshi and E. Collet, Physical Review B 102 (13), $134104(2020)$.

26. M. Shatruk, H. Phan, B. A. Chrisostomo and A. Suleimenova, Coordination Chemistry Reviews 289-290, 62-73 (2015).

27. E. Trzop, D. Zhang, L. Pineiro-Lopez, F. J. Valverde-Munoz, M. Carmen Munoz, L. Palatinus, L. Guerin, H. Cailleau, J. A. Real and E. Collet, Angew Chem Int Ed Engl 55 (30), 8675-8679 (2016).

28. E. Collet and P. Guionneau, Cr Chim 21 (12), 1133-1151 (2018).

29. P. Guionneau, Dalton Transactions 43 (2), 382-393 (2014). 
30. H. Watanabe, K. Tanaka, N. Bréfuel, H. Cailleau, J.-F. Létard, S. Ravy, P. Fertey, M. Nishino, S. Miyashita and E. Collet, Physical Review B 93, 014419 (2016).

31. M. Paez-Espejo, M. Sy and K. Boukheddaden, J Am Chem Soc 138 (9), 3202-3210 (2016).

32. C. Enachescu, M. Nishino, S. Miyashita, K. Boukheddaden, F. Varret and P. A. Rikvold, Physical Review B 91 (10), 104102 (2015).

33. K. Boukheddaden, I. Shteto, B. Hôo and F. Varret, Physical Review B 62 (22), 14806-14817 (2000).

34. W. Nicolazzi and A. Bousseksou, Cr Chim 21 (12), 1060-1074 (2018).

35. D. Chernyshov, H.-B. Bürgi, M. Hostettler and K. W. Törnroos, Physical Review B 70, 094116 (2004).

36. J. F. Letard, P. Guionneau, L. Rabardel, J. A. K. Howard, A. E. Goeta, D. Chasseau and O. Kahn, Inorganic Chemistry 37 (17), 4432-4441 (1998).

37. P. Guionneau, J. F. Letard, D. S. Yufit, D. Chasseau, G. Bravic, A. E. Goeta, J. A. K. Howard and O. Kahn, Journal of Materials Chemistry 9 (4), 985-994 (1999).

38. J. F. Letard, G. Chastanet, O. Nguyen, S. Marcen, M. Marchivie, P. Guionneau, D. Chasseau and P. Gutlich, Monatshefte Fur Chemie 134 (2), 165-182 (2003).

39. K. Ichiyanagi, J. Hebert, L. Toupet, H. Cailleau, P. Guionneau, J. F. Létard and E. Collet, Physical Review B 73 (6) (2006).

40. M. Buron-Le Cointe, J. Hébert, C. Baldé, N. Moisan, L. Toupet, P. Guionneau, J. F. Létard, E. Freysz, H. Cailleau and E. Collet, Physical Review B 85, 064114 (2012).

41. J. Zarembowitch, F. Varret, A. Hauser, J.-A. Real and K. Boukheddaden, Cr Chim 21 (12), 1056-1059 (2018).

42. E. K. Salje, Phase Transitions in Ferroelastic and Co-elastic Crystals. (Cambridge University Press, Cambridge, 1991).

43. L. Landau, Ukr. J. Phys. 53, 25-35 (2008).

44. E. Collet, L. Henry, L. Piñeiro-López, L. Toupet and J. A. Real, Current Inorganic Chemistry 6 (1), 61-66 (2016).

45. S. Ohkoshi, T. Matsuda, H. Tokoro and K. Hashimoto, Chem Mater 17 (1), 81-84 (2005).

46. H. Tokoro, S. Miyashita, K. Hashimoto and S. Ohkoshi, Physical Review B 73, 172415 (2006).

47. K. Boukheddaden, E. D. Loutete-Dangui, E. Codjovi, M. Castro, J. A. Rodriguéz-Velamazán, S. Ohkoshi, H. Tokoro, M. Koubaa, Y. Abid and F. Varret, Journal of Applied Physics 109 (1), 013520 (2011).

48. V. B. Jakobsen, E. Trzop, L. C. Gavin, E. Dobbelaar, S. Chikara, X. Ding, K. Esien, H. Müller-Bunz, S. Felton, V. S. Zapf, E. Collet, M. A. Carpenter and G. G. Morgan, Angewandte Chemie International Edition 59 (32), 13305-13312 (2020).

49. H. Watanabe, N. Brefuel, E. Collet, L. Toupet, K. Tanaka and J. P. Tuchagues, Eur J Inorg Chem (5-6), 710-715 (2013).

50. M. G. Cowan, J. Olguín, S. Narayanaswamy, J. L. Tallon and S. Brooker, Journal of the American Chemical Society 134 (6), 2892-2894 (2012).

51. V. Janovec and J. Privratska, International Tables for Crystallography D, 449-505 (2006).

52. V. Janovec, V. Dvořák and J. Petzelt, Czechoslovak Journal of Physics B 25 (12), 1362-1396 (1975).

53. A. Marino, P. Chakraborty, M. Servol, M. Lorenc, E. Collet and A. Hauser, Angewandte ChemieInternational Edition 53 (15), 3863-3867 (2014).

54. J. Kusz, M. Zubko, R. B. Neder and P. Gutlich, Acta Crystallographica Section B 68 (1), 40-56 (2012).

55. C. Chong, H. Mishra, K. Boukheddaden, S. Denise, G. Bouchez, E. Collet, J. C. Ameline, A. D. Naik, Y. Garcia and F. Varret, J Phys Chem B 114 (5), 1975-1984 (2010).

56. A. Goujon, F. Varret, K. Boukheddaden, C. Chong, J. Jeftić, Y. Garcia, A. D. Naik, J. C. Ameline and E. Collet, Inorganica Chimica Acta 361 (14-15), 4055-4064 (2008).

57. A. Hauser, Chemical Physics Letters 192 (1), 65-70 (1992).

58. A. Hauser, Journal of Chemical Physics 94 (4), 2741-2748 (1991).

59. M.-H. Lemée-Cailleau, C. Ecolivet, B. Ouladdiaf, F. Moussa and J.-F. Létard, Physica B: Condensed Matter 404 (3-4), 379-381 (2009).

60. M. H. Lemée-Cailleau, C. Ecolivet, B. Ouladdiaf, F. Moussa, J. Jeftic and J. F. Létard, J Magn Magn Mater 310 (2, Part 2), 1792-1793 (2007).

61. Y. Garcia, O. Kahn, L. Rabardel, B. Chansou, L. Salmon and J. P. Tuchagues, Inorganic Chemistry 38 (21), 4663-4670 (1999).

62. B. Weber, C. Carbonera, C. Desplances and J.-F. Létard, Eur J Inorg Chem 2008 (10), 1589-1598 (2008). 
63. Galina S. Matouzenko, J.-F. Létard, S. Lecocq, A. Bousseksou, L. Capes, L. Salmon, M. Perrin, O. Kahn and A. Collet, Eur J Inorg Chem 2001 (11), 2935-2945 (2001).

64. E. Milin, V. Patinec, S. Triki, E.-E. Bendeif, S. Pillet, M. Marchivie, G. Chastanet and K. Boukheddaden, Inorganic Chemistry 55 (22), 11652-11661 (2016).

65. T. Boonprab, S. J. Lee, S. G. Telfer, K. S. Murray, W. Phonsri, G. Chastanet, E. Collet, E. Trzop, G. N. L. Jameson, P. Harding and D. J. Harding, Angew Chem Int Ed Engl 58 (34), 11811-11815 (2019).

66. R. Traiche, M. Sy and K. Boukheddaden, The Journal of Physical Chemistry C 122 (7), 4083-4096 (2018).

67. N. Brefuel, H. Watanabe, L. Toupet, J. Come, N. Matsumoto, E. Collet, K. Tanaka and J. P. Tuchagues, Angew Chem Int Ed Engl 48 (49), 9304-9307 (2009).

68. N. Brefuel, E. Collet, H. Watanabe, M. Kojima, N. Matsumoto, L. Toupet, K. Tanaka and J. P. Tuchagues, Chemistry 16 (47), 14060-14068 (2010).

69. S. Hayami, Y. Komatsu, T. Shimizu, H. Kamihata and Y. H. Lee, Coordination Chemistry Reviews 255 (17), 1981-1990 (2011).

70. D. Boinnard, A. Bousseksou, A. Dworkin, J. M. Savariault, F. Varret and J. P. Tuchagues, Inorganic Chemistry 33 (2), 271-281 (1994).

71. D. Chernyshov, M. Hostettler, K. W. Tornroos and H. B. Burgi, Angew Chem Int Ed Engl 42 (32), 38253830 (2003).

72. D. L. Reger, C. A. Little, V. G. Young and M. Pink, Inorganic Chemistry 40 (12), 2870-2874 (2001).

73. V. A. Money, C. Carbonera, J. Elhaïk, M. A. Halcrow, J. A. K. Howard and J.-F. Létard, Chemistry - A European Journal 13 (19), 5503-5514 (2007).

74. J. Luan, J. Zhou, Z. Liu, B. Zhu, H. Wang, X. Bao, W. Liu, M.-L. Tong, G. Peng, H. Peng, L. Salmon and A. Bousseksou, Inorganic Chemistry 54 (11), 5145-5147 (2015).

75. M. Yamada, H. Hagiwara, H. Torigoe, N. Matsumoto, M. Kojima, F. Dahan, J.-P. Tuchagues, N. Re and S. Iijima, Chemistry - A European Journal 12 (17), 4536-4549 (2006).

76. T. Sato, K. Nishi, S. Iijima, M. Kojima and N. Matsumoto, Inorganic Chemistry 48 (15), 7211-7229 (2009).

77. S. Bonnet, M. A. Siegler, J. S. Costa, G. Molnar, A. Bousseksou, A. L. Spek, P. Gamez and J. Reedijk, Chem Commun (43), 5619-5621 (2008).

78. N. Ortega-Villar, C. M. Muñoz and A. J. Real, Magnetochemistry 2 (1) (2016).

79. M. Nishino and S. Miyashita, Physical Review B 88 (1), 014108 (2013).

80. M. Nishino, C. Enachescu and S. Miyashita, Physical Review B 100 (13), 134414 (2019).

81. N. Huby, L. Guérin, E. Collet, L. Toupet, J.-C. Ameline, H. Cailleau, T. Roisnel, T. Tayagaki and K. Tanaka, Physical Review B 69 (2), 020101 (2004).

82. A. Marino, M. Buron-Le Cointe, M. Lorenc, L. Toupet, R. Henning, A. D. DiChiara, K. Moffat, N. Brefuel and E. Collet, Faraday Discuss 177, 363-379 (2015).

83. P. Bak and R. Bruinsma, Physical Review Letters 49 (4), 249-251 (1982).

84. B. Weber, W. Bauer and J. Obel, Angewandte Chemie International Edition 47 (52), 10098-10101 (2008).

85. S. Pillet, E.-E. Bendeif, S. Bonnet, H. J. Shepherd and P. Guionneau, Physical Review B 86 (6), 064106 (2012).

86. J. E. Clements, J. R. Price, S. M. Neville and C. J. Kepert, Angew Chem Int Ed Engl 55 (48), 1510515109 (2016).

87. J. Cruddas and B. J. Powell, Inorganic Chemistry Frontiers 7 (22), 4424-4437 (2020).

88. E. Collet, H. Watanabe, N. Brefuel, L. Palatinus, L. Roudaut, L. Toupet, K. Tanaka, J. P. Tuchagues, P. Fertey, S. Ravy, B. Toudic and H. Cailleau, Phys Rev Lett 109 (25), 257206 (2012). 


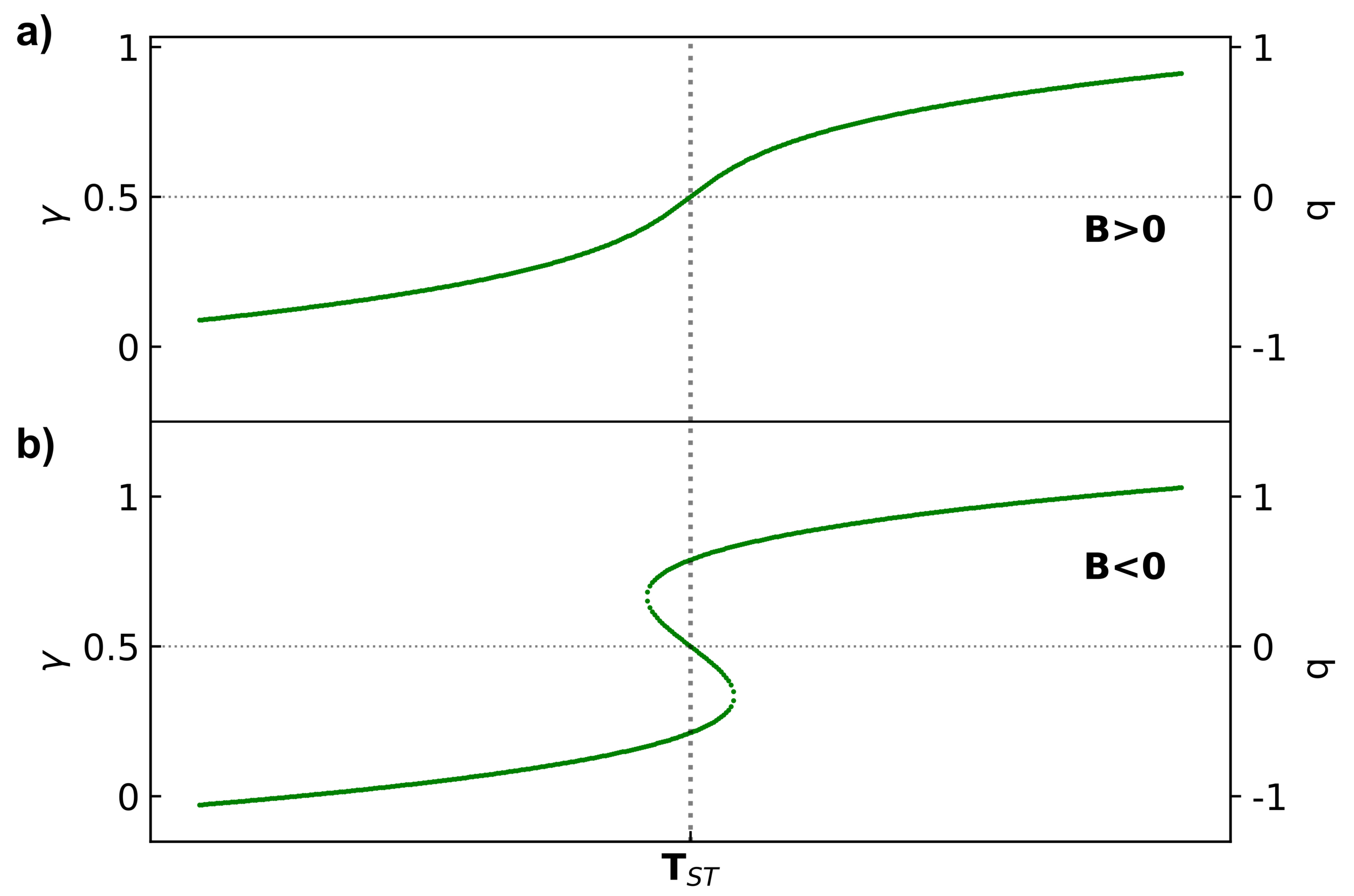



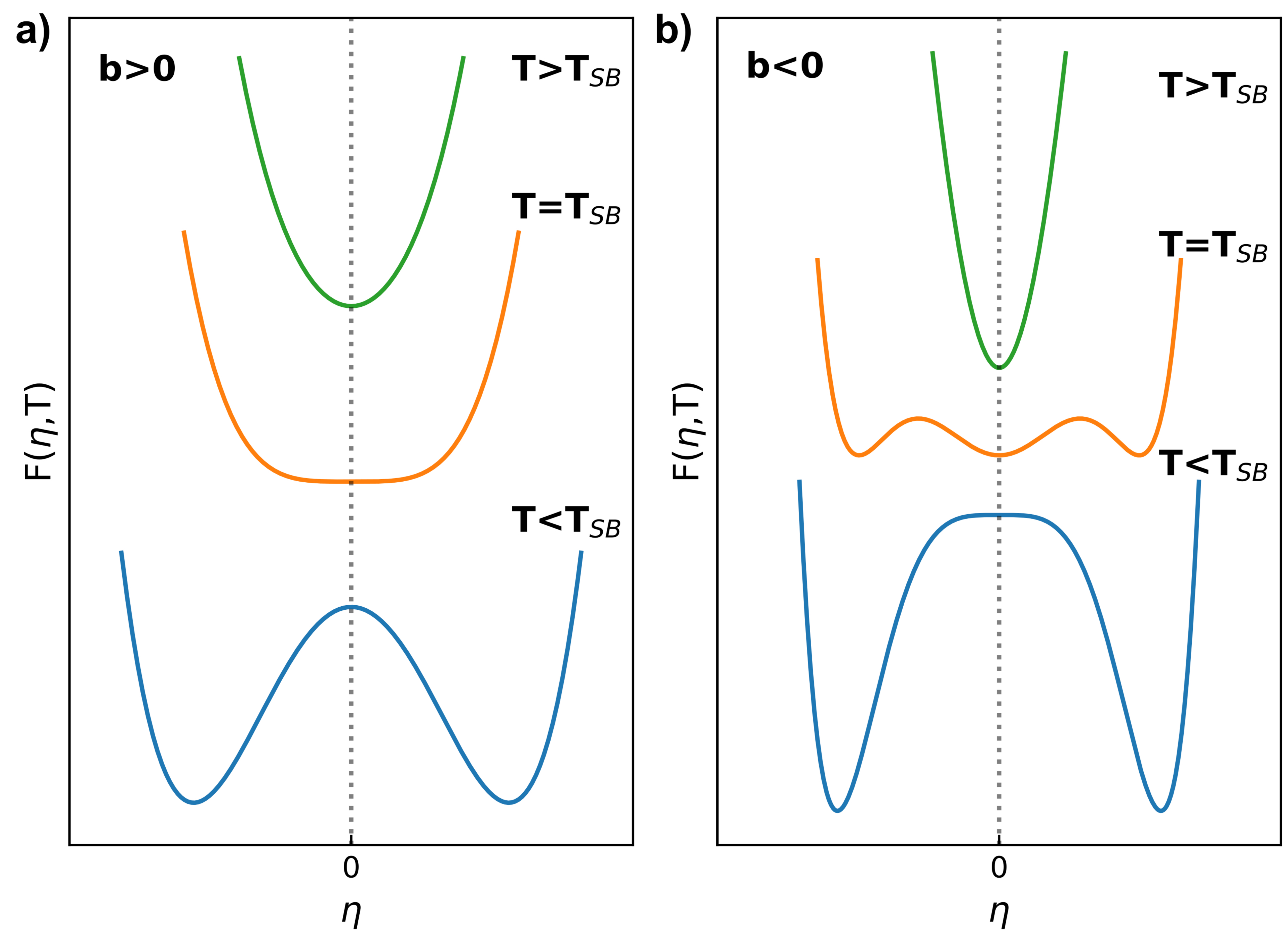


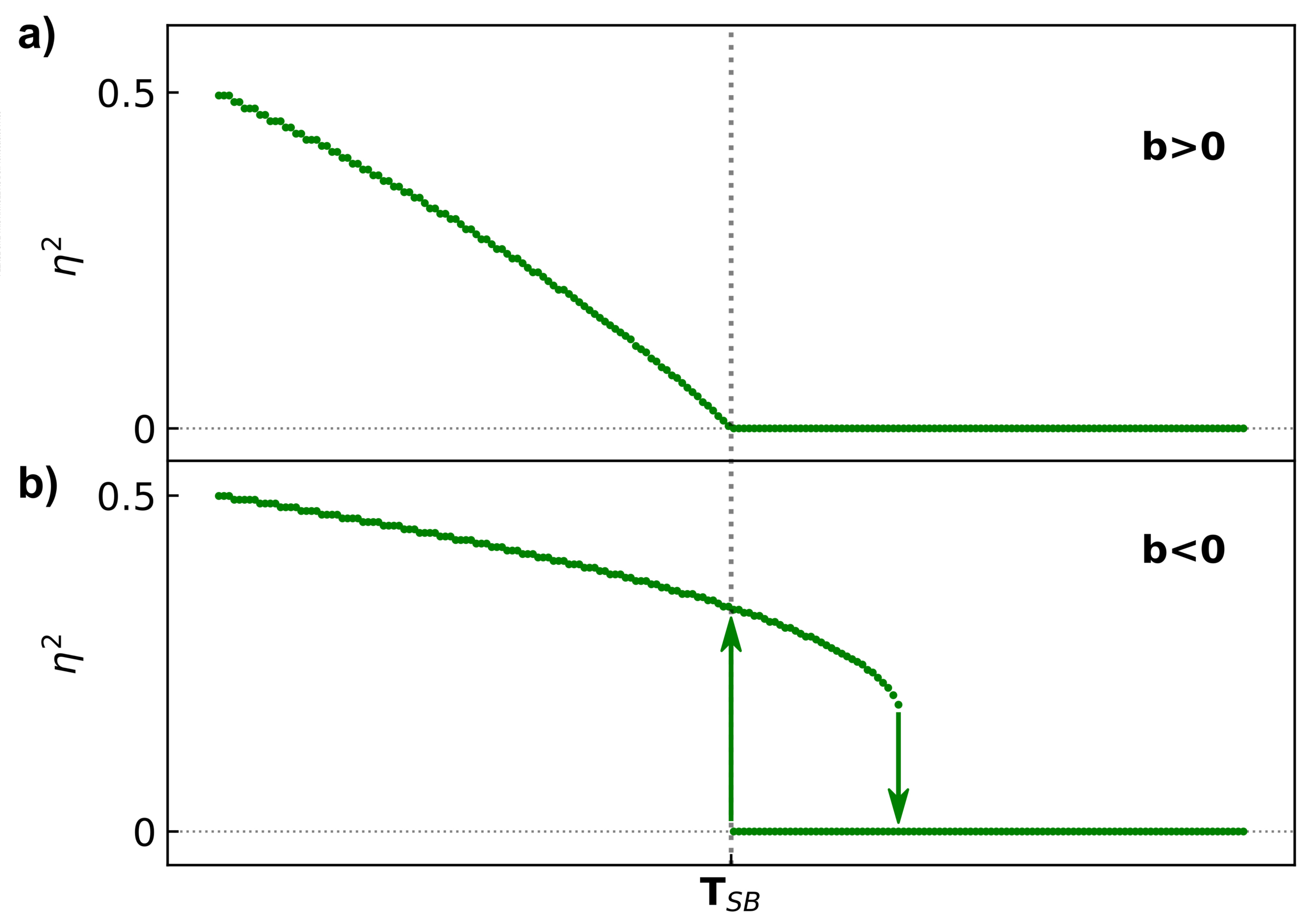




\section{b $<0 \quad B<0$}

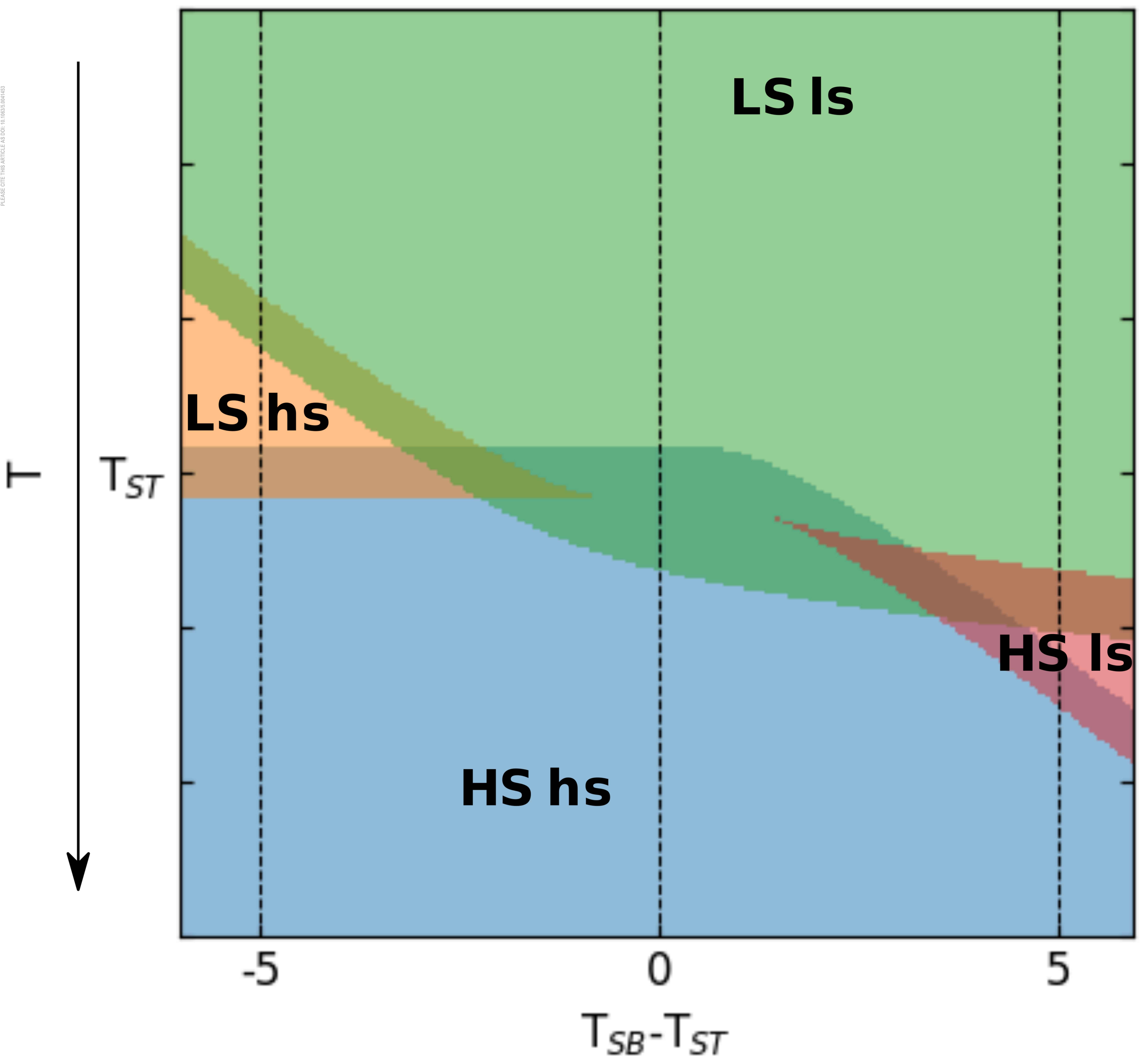




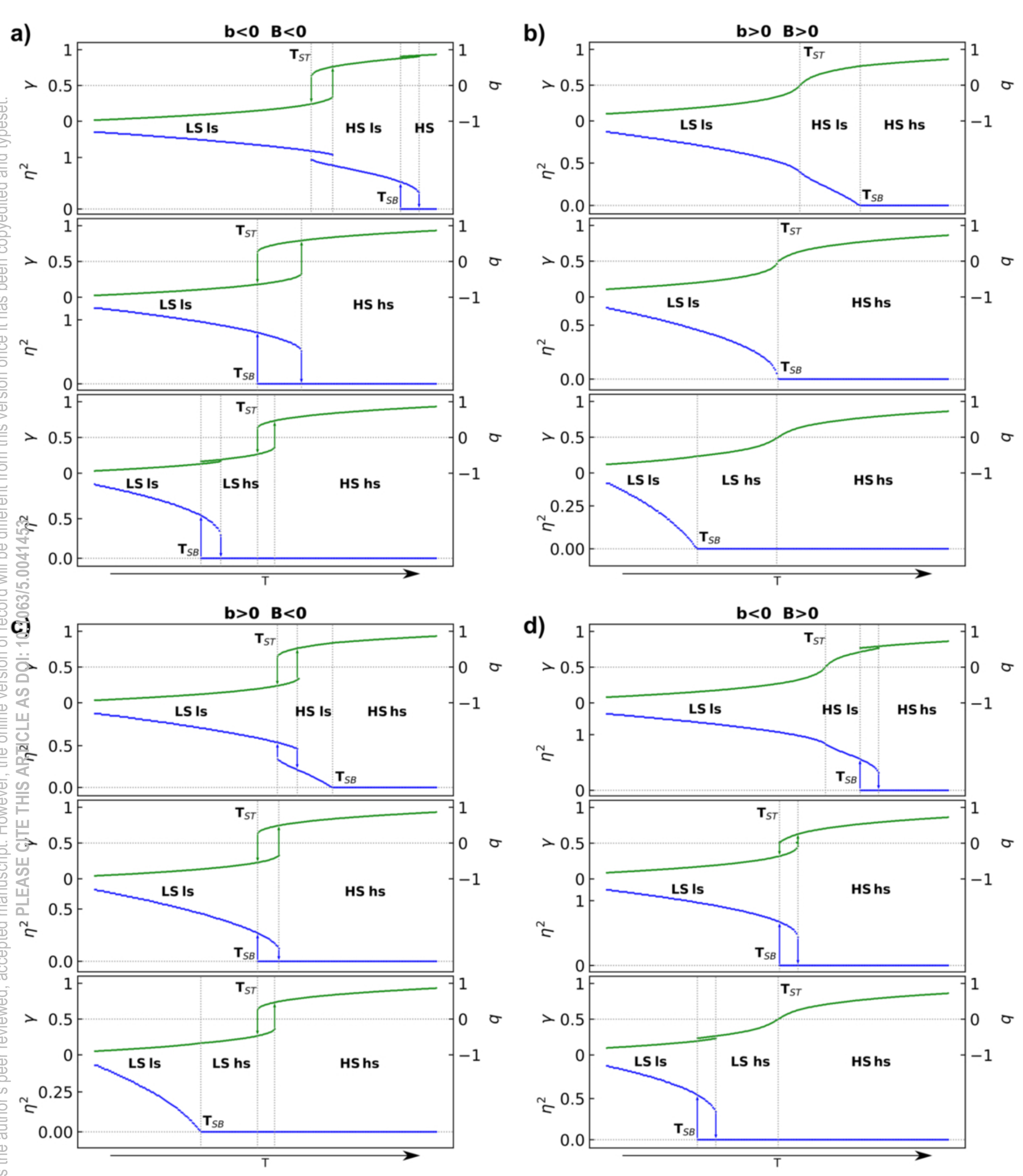


LSIs

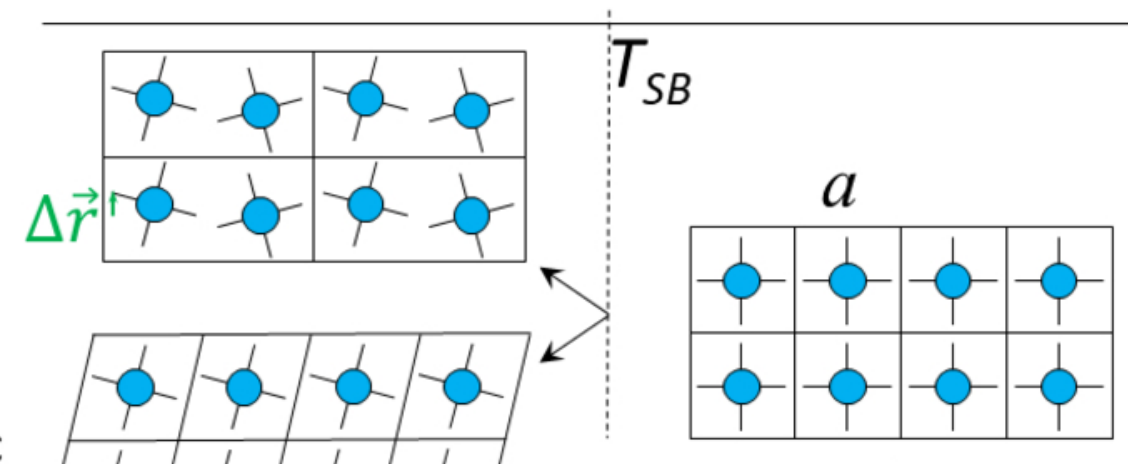
distortion doubling

LSIS

Ferroelastic

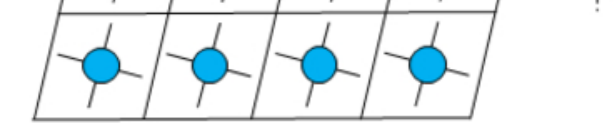

LShs
$T_{S T}$

a

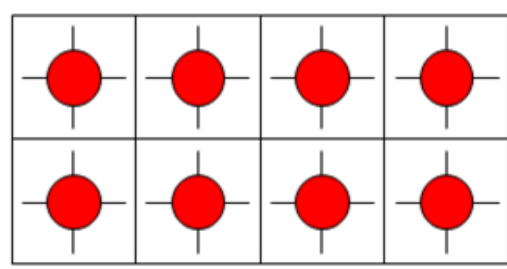

HShs

$\vec{T}$ s 


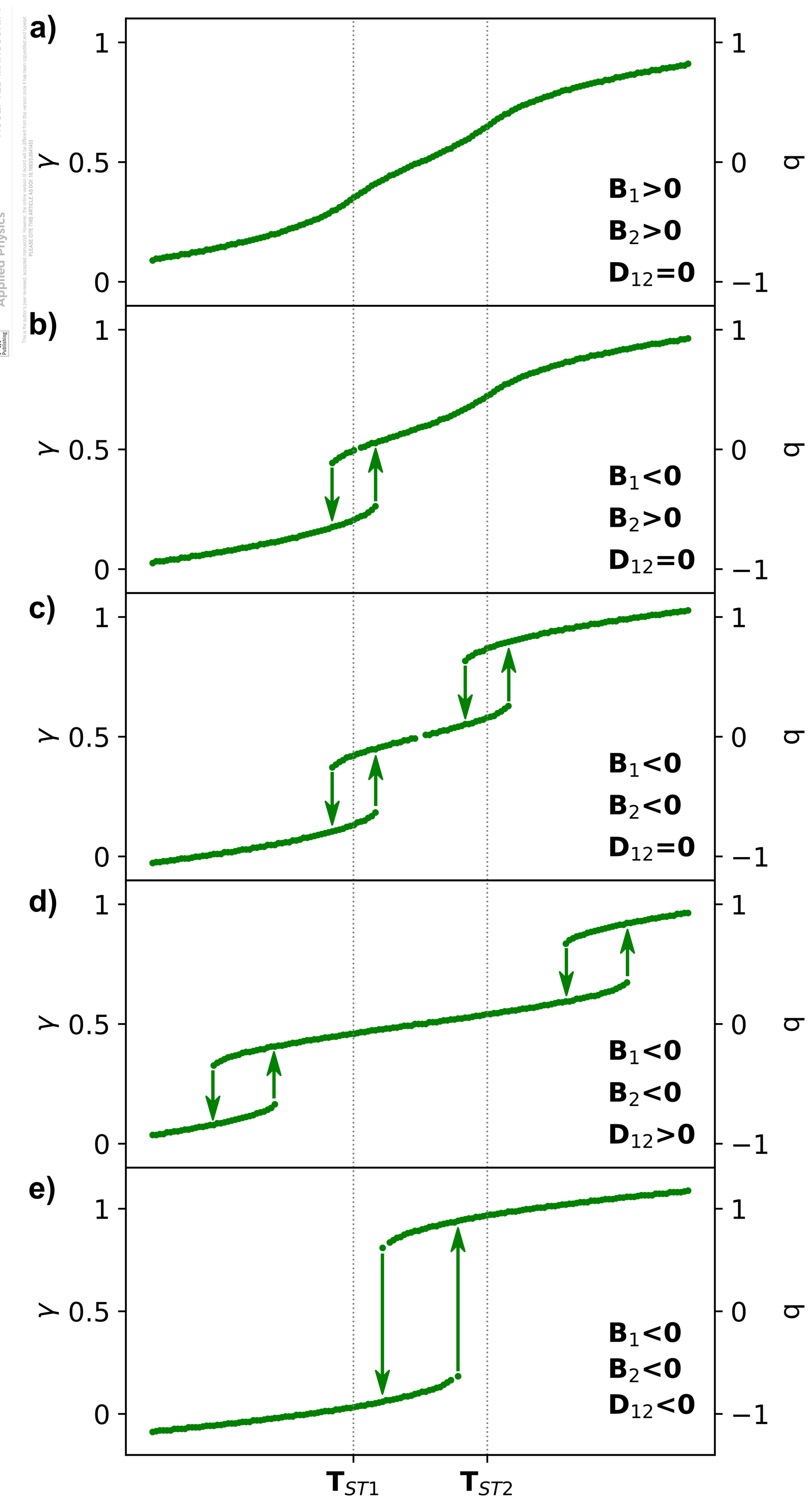


a) Non-symmetry-breaking stepwise

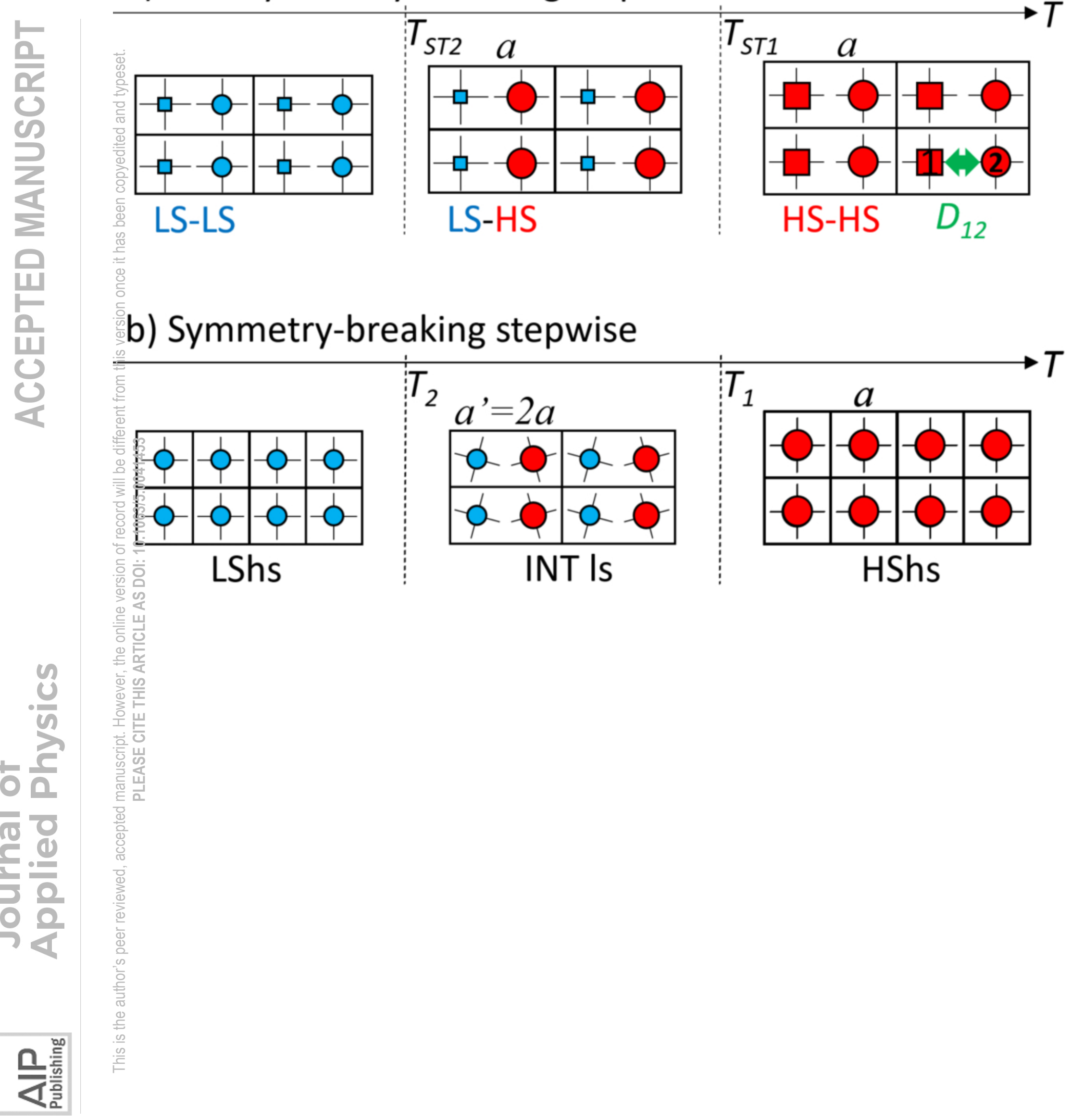




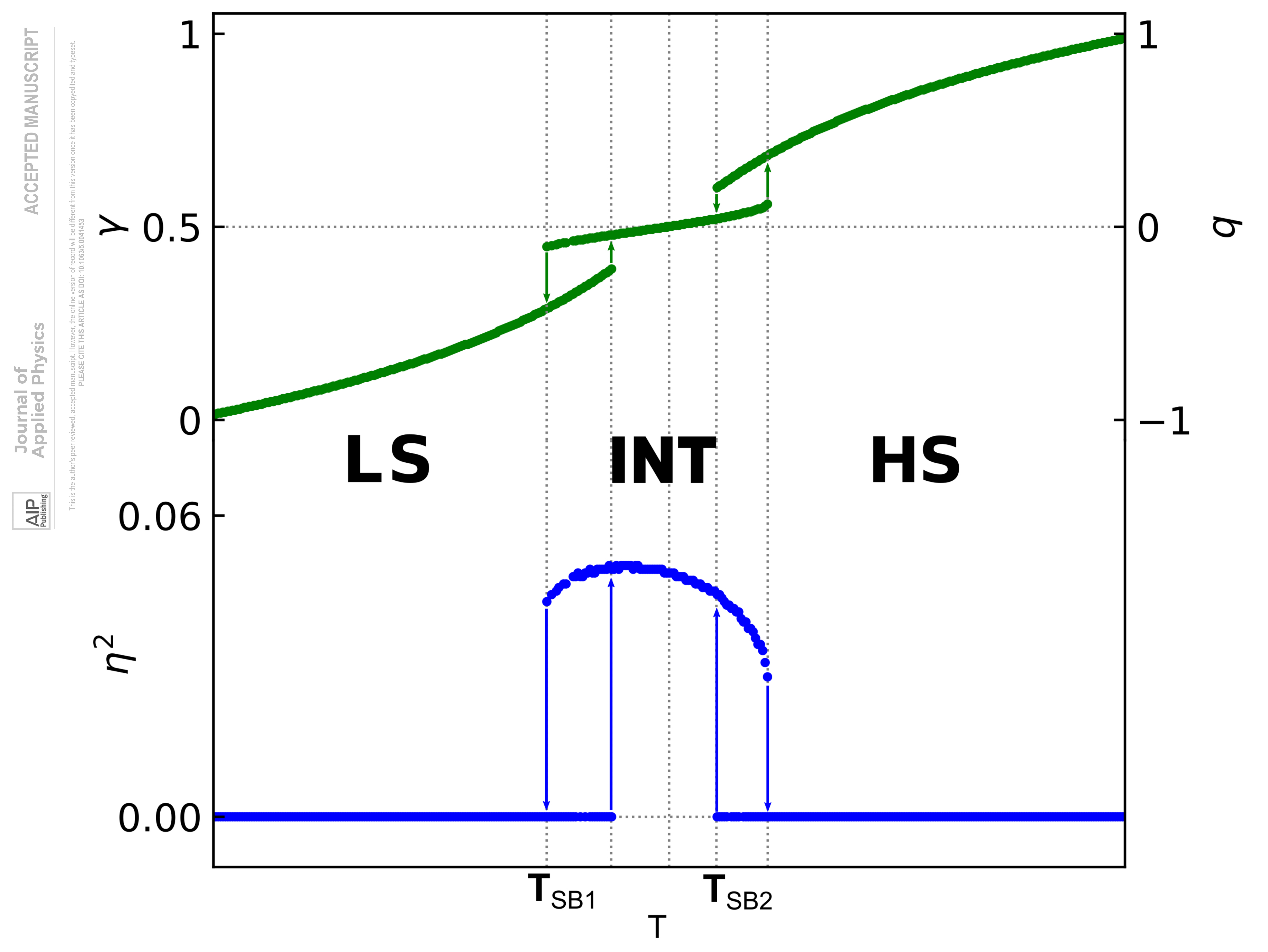


Z LS $100 \mathrm{~K} \mathrm{PC}$ if HS

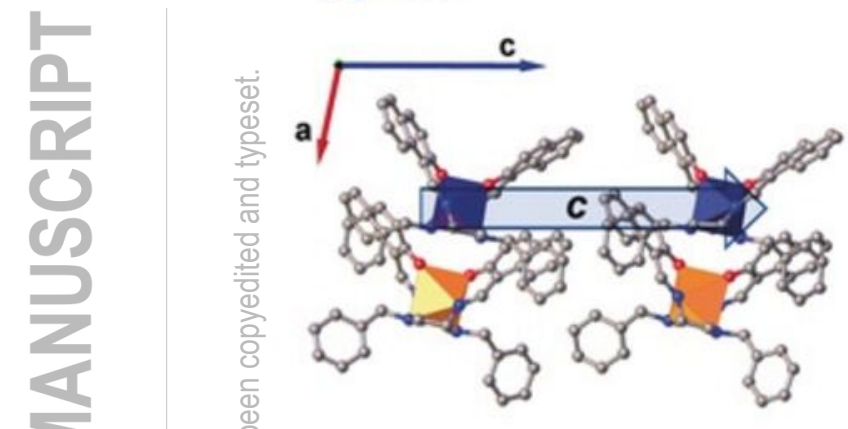

$\nabla$ HS-Fe2 $150 \mathrm{~K} \mathrm{Pn}$ … HS-Fe1

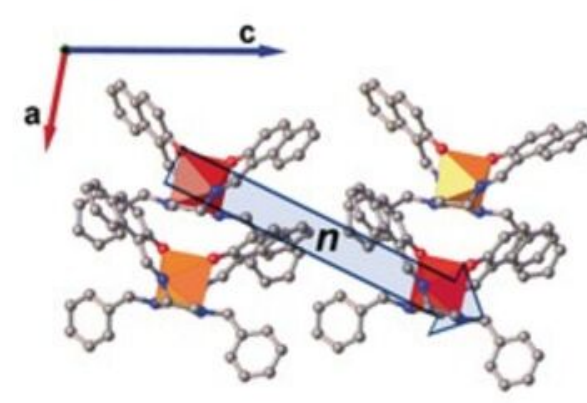

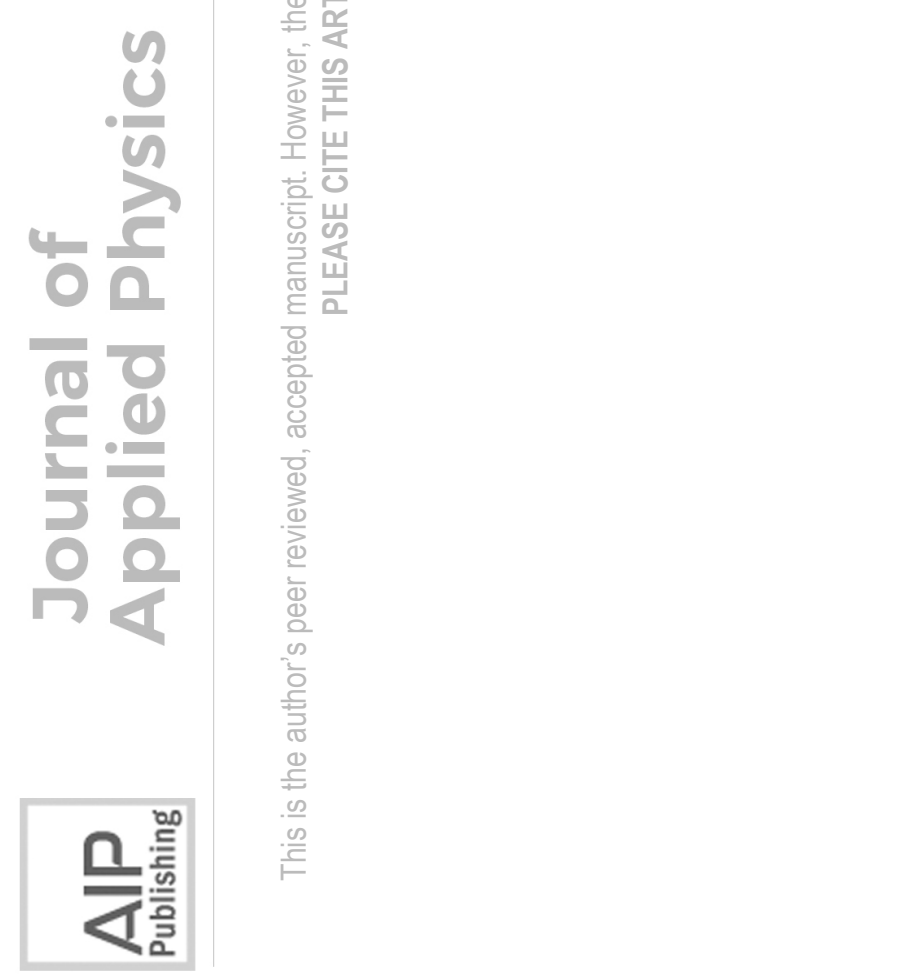

